ApJ, 567, 828 (2002); e-print astro-ph/0103333

\title{
SPECTRA AND GROWTH RATES OF FLUCTUATING MAGNETIC FIELDS IN THE KINEMATIC DYNAMO THEORY WITH LARGE MAGNETIC PRANDTL NUMBERS
}

\author{
Alexander A. Schekochihin ${ }^{1}$ \\ Plasma Physics Laboratory, Princeton University, P. O. Box 451, Princeton, New Jersey 08543 \\ sure@pppl.gov \\ Stanislav A. Boldyrev \\ Institute for Theoretical Physics, University of California, Santa Barbara, California 93106 \\ boldyrev@itp.ucsb.edu \\ and \\ Russell M. Kulsrud \\ Princeton University Observatory, Peyton Hall, Princeton, New Jersey 08544 \\ rkulsrud@astro.princeton.edu \\ 6 March 2001; revised 21 September 2001
}

\begin{abstract}
The existence of a weak galactic magnetic field has been repeatedly confirmed by observational data. The origin of this field has not as yet been explained in a fully satisfactory way and represents one of the main challenges of the astrophysical dynamo theory. In both the galactic dynamo theory and the primordial-origin theory, a major influence is exerted by the small-scale magnetic fluctuations. This article is devoted to constructing a systematic second-order statistical theory of such small-scale fields. The statistics of these fields are studied in the kinematic approximation and for the case of large Prandtl numbers, which is relevant for the galactic and protogalactic plasma. The advecting velocity field is assumed to be Gaussian and short-time correlated. Theoretical understanding of this kinematic dynamo model is a necessary prerequisite for any prospective nonlinear dynamo theory. The theory is developed for an arbitrary degree of compressibility and formally in $d$ dimensions, which generalizes the previously known results, elicits the structure of the solutions, and uncovers a number of new effects. The magnetic energy spectra are studied as they grow and spread over scales during the initial stage of the field amplification. Exact Green's-function solutions are obtained. The spectral theory is supplemented by the study of magnetic-field correlation functions in the configuration space, where the dynamo problem can be mapped onto a particular one-dimensional quantum-mechanical problem. The latter approach is most suitable for the description of the kinematic dynamo in the long-time limit, i.e. when the magnetic excitation has spread over all scales present in the system. A simple way of calculating the growth rates of the magnetic fields in this long-time limit is proposed.
\end{abstract}

\footnotetext{
${ }^{1}$ Present address: Imperial College, Blackett Laboratory, Prince Consort Rd, London SW7 2BW, U.K.
} 
Subject headings: galaxies: magnetic fields - ISM: magnetic fields — magnetic fields — methods: analytical — MHD — turbulence

\section{INTRODUCTION}

\subsection{Astrophysical Motivation}

The question of the origin of the galactic magnetic field has long been a subject of much interest in plasma astrophysics. The existence of galactic magnetic fields was first inferred by Alfvén (1937a,b) and Fermi (1949) from the properties of cosmic rays. This was later confirmed by observational data (Hiltner 1949; Hall and Mikesell 1949). Modern observations indicate that our Galaxy possesses a magnetic field that has a large-scale component that is several $\mu \mathrm{G}$ strong and is coherent on the scales of up to a kiloparsec $\left(\sim 10^{21} \mathrm{~cm}\right)$. These scales are intermediate between the diameter of the galactic disk $(\sim 10 \mathrm{kpc})$ and its thickness $(\sim 100 \mathrm{pc})$. Observations indicate that magnetic fields of similar magnitudes (which correspond to magnetic-energy densities comparable to those of the fluid motions of the interstellar matter) and spatial coherence are common in other galaxies as well (see reviews by Kronberg 1994; Beck et al. 1996; Zweibel and Heiles 1997). Most of the theories that have been advanced to explain the presence of these fields have in one way or another connected their origin with the dynamo action of the interstellar turbulence.

The interstellar medium (ISM) consists of a partially ionized plasma that is regularly stirred on the scale of about 100 parsec by the shock waves generated by supernova explosions. The Reynolds number of the ISM is of the order of $10^{5}$, which allows a fully developed Kolmogorov-type turbulent cascade to be set up. The energy generated at the outer scale of $\sim 100$ parsec is thus transfered approximately four decades down to the Kolmogorov inner scale, where it is dissipated by the molecular viscosity. If an initial seed magnetic field is introduced into such a medium, the turbulent velocity field should be expected to stretch the magnetic-field lines and thus amplify the field via the usual fast-dynamo mechanism (Sakharov 1982; Vainshtein and Zeldovich 1972). The turbulent nature of the physical processes involved clearly necessitates a statistical description.

Traditionally, the hopes for a theoretical explanation of the galactic magnetic field have focused upon the mean-field dynamo theories (Parker 1955; Braginskii 1965b; Steenbeck, Krause, and Rädler 1966; Moffatt 1978; Parker 1979; Ruzmaikin, Shukurov, and Sokoloff 1988). The essential idea is to follow the evolution of the volume-averaged (i.e., effectively, large-scale) magnetic field subject to two main assumptions. First, it is assumed that the initial seed field is small and thus, during the initial stage of its evolution, the magnetic field is too weak to exert a significant amount of back reaction on the hydrodynamic motions. The Lorentz forces can therefore be neglected and magnetic field considered passive, which gives rise to the so-called kinematic approximation. Second, the large-scale mean field is assumed to be much stronger than the small-scale magnetic fluctuations. ${ }^{2}$ It turns out that, if these two assumptions hold, and if the interstellar turbulence lacks mirror invariance, the mean field will grow exponentially at a rate proportional to the amount of helicity possessed by the turbulent medium. Such mean-field amplification has come to be referred to as the $\alpha$ effect.

It must, however, be appreciated that the mean-field theory faces serious challenges to its theoretical and physical foundations. It was pointed out already by Batchelor (1950) that the initial amplification of

\footnotetext{
${ }^{2}$ This assumption can be circumvented for the Gaussian $\delta$-correlated velocity field discussed in $\S 1.2$ (Vainshtein 1970; Kulsrud and Anderson 1992; Boldyrev 2001).
} 
the magnetic field in the kinematic regime would be accompanied by the transfer of the magnetic energy to small (nonhydrodynamic) scales. A wide range of such scales exists as a result of the huge disparity between the hydrodynamic and the magnetic Reynolds numbers of the ISM: while Re $\sim 10^{5}$, its magnetic counterpart can be as high as $\mathrm{R}_{m} \sim 10^{19}$. The ratio of these two numbers, commonly referred to as the magnetic Prandtl number, $\operatorname{Pr} \sim \mathrm{R}_{m} / \mathrm{Re}$, determines the width of the scale interval between the Kolmogorov inner scale $k_{\nu}$, where the viscous dissipation cuts off the hydrodynamic turbulence spectrum, and the resistive scale $k_{\eta}$, where magnetic energy is dissipated by the Ohmic resistive-diffusive mechanism. Since $k_{\eta} / k_{\nu} \sim \operatorname{Pr}^{1 / 2}$, the subviscous scales accessible to the small-scale fluctuating magnetic fields extend over as many as seven decades in the wave-number space. ${ }^{3}$ In the kinematic approximation, the rates of the exponential growth of the small-scale magnetic-fluctuation energy and of its transfer toward the small scales turn out to greatly (by the factor of $\sim 10^{4}$ ) exceed the growth rate of the mean field due to the $\alpha$-effect, which operates on a time scale associated with the overall galactic rotation. Unlike the mean field, the magnetic energy grows regardless and independently of whether the turbulent velocity field has a helical component. Thus, the energy of the small-scale magnetic fluctuations must be expected to grow to equipartition with that of the smallest eddies of the turbulent velocity field much faster than the mean field can reach any appreciable values. The validity of the kinematic mean-field theory will therefore break down long before the growth mechanism predicted by it has time to manifest itself (Kulsrud and Anderson 1992).

The mean-field approach is an attempt to explain the large-scale galactic magnetic field in terms of a coherent volume-averaged field being amplified by the hydrodynamic helicity effect. Besides the impossibility to neglect the small-scale fields, there exists another physical consideration that makes justifying this view rather problematic. Since no magnetic-energy dissipation mechanism is available at the large scales, the growth of the large-scale field must be consistent with the flux-conservation constraint. The most popular theory has been that the magnetic-field lines are partly expelled from the galactic disk (see Parker 1979; Kulsrud 1999, 2000). However, the possibility of flux expulsion has been increasingly in doubt (Rafikov and Kulsrud 2000).

In view of the fundamental difficulties associated with the mean-field approach, one is hard-pressed to seek alternative ways to construct an adequate galactic dynamo theory. A promising avenue of investigation naturally presents itself in the context of the small-scale-field amplification. It seems quite reasonably clear and has been an accepted point of view since the work of Batchelor (1950) that the growth of the fluctuating fields at subviscous scales should culminate in the magnetic-fluctuation energy equalizing with that of the smallest turbulent eddies. What happens next is a subject of much interest and disagreement. The basic point of contention is whether the magnetic energy would saturate at viscous (Batchelor 1950) or resistive (Vainshtein and Cattaneo 1992; Gruzinov and Diamond 1994, 1995, 1996) scales, or rather proceed to reach full equipartition with the turbulence (Biermann and Schlüter 1951).

An explanation of the existence of the large-scale galactic magnetic field could be within grasp if it were to be demonstrated that, once the magnetic-fluctuation energy had equalized with that of the smallest eddies, an inverse cascade would take place, forcing the magnetic excitation to keep growing and to gradually move towards ever-larger scales until equipartition were achieved at all scales up to the energy-containing ones. A statistical theory of this sort would predict the emergence of large-scale magnetic fluctuations with energy comparable to that of the turbulence (see Sec. 4 for further discussion of these matters). From the point of view of the mean-field approach, these fluctuations would still have a zero statistical average. However,

\footnotetext{
${ }^{3}$ In this context, it should be noted that resolving such a broad range of scales in a numerical simulation is all but impossible, so theoretical understanding of the physics of the small-scale fields is indispensable.
} 
observationally, they would locally appear as a field coherent at large scales (cf. Blackman 1998).

This idea faces a serious setback when measured against the actual parameters of the ISM. Namely, the typical energy-containing scales of the galactic turbulence, to which one can expect the inverse cascade to bring the magnetic energy, are of the order of 100 parsec (the average distance between the supernovae, which drive the turbulence), or about 10 times smaller than the coherence scale of the observed large-scale galactic magnetic field. This problem can be resolved if one follows Kulsrud et al. (1997a) in their recent suggestion to consider the possibility of a primordial origin of the galactic magnetic field. The primordial-origin hypothesis regards the large-scale galactic field not as a product of galactic turbulent dynamo mechanisms, but as a residue of the analogous processes that take place in protogalaxies before they collapse into galaxies.

Indeed, the conditions in the protogalactic plasma cloud seem to be more favorable than those in the galactic ISM for the operation of a bona fide turbulent dynamo. As does the ISM, the protogalactic plasma supports a Kolmogorov-type turbulence, this time driven by the shock waves originating from the instabilities associated with the forces of gravitational collapse. These instabilities occur on the scales comparable to the size of the protogalaxy ( $\sim 100$ kiloparsec) and thus, unlike galaxies, protogalaxies know no disparity between the system size and the energy-containing scale of the turbulence. The hydrodynamic Reynolds number of the protogalactic plasma is $\operatorname{Re} \sim 10^{4}$, so an inertial range about three decades wide is available for the Kolmogorov turbulent energy cascade. Numerical simulations indicate that such a cascade is indeed set up (Kulsrud et al. 1997a). The protogalactic Prandtl number is even larger than the galactic one: $\operatorname{Pr} \sim 10^{22}$, which allows as many as eleven decades of subviscous scales accessible to the magnetic fields.

The seed magnetic fields in the protogalaxy could be created from an initial state with no fields at all by the so-called Biermann-battery mechanism (Biermann 1950; Kulsrud et al. 1997a). This battery action is associated with the thermoelectric term in the plasma Ohm's law. This term is non-zero provided the motions are nonbarotropic $(\nabla p \times \nabla \rho \neq 0)$. Since the violent beginnings of the protogalaxy may be assumed to allow nonbarotropic large-scale pressure fluctuations, it can be estimated that the battery is capable of generating large-scale magnetic fields of about $10^{-21} \mathrm{G}$ before the dynamo term becomes dominant.

The magnetic fields are then amplified and carried over to small scales by the same Batchelor (1950) mechanism that foiled the galactic mean-field theory. After the equipartition with the smallest turbulent eddies is reached, an inverse cascade may be envisioned that brings the magnetic energy back to the large scales. As the protogalaxy collapses into the galactic disk, its magnetic field is compressed and becomes the initial magnetic field of the newly formed galaxy. If the pre-collapse protogalactic magnetic field is amplified by the protogalactic turbulence to sufficiently large values, it may well be enough to give rise, upon compression, to a galactic field with the strength and scale of coherence reasonably close to those observed (Howard and Kulsrud 1997; Kulsrud et al. 1997b; Kulsrud 1999, 2000). [A midway approach is to consider the residual field resulting from the protogalactic dynamo as the seed field for further operation of the galactic dynamo mechanisms such as, e.g, the $\alpha$ - $\Omega$ dynamo (Parker 1979; Kulsrud 1999, 2000) (see also Blackman 1998).] While this scenario is certainly largely hypothetical, it offers a physical possibility that can hardly be neglected.

In all of the above discussion, the fluctuating magnetic fields in general and the small-scale fields in particular have played a prominent role. Under additional assumptions about the turbulent velocity field, the kinematic stage of their evolution can be treated analytically and exactly. Any theoretical understanding of the processes that occur once the nonlinear stage is initiated, must rely on a solid statistical theory of the small-scale kinematic dynamo. In this paper, we give a detailed and systematic exposition of the smallscale kinematic dynamo theory, following an often-neglected Cartesian principle: " ... je me persuadai ... 
que, pour toutes les opinions que j'avais reçues jusques alors en ma créance, je ne pouvais mieux faire que d'entreprendre une bonne foi de les en ôter, afin d'y en remettre par après, ou d'autres meilleures, ou bien les mêmes, lorsque je les aurais ajustées au niveau de la raison"(Descartes 1637). ${ }^{4}$

\subsection{The Kinematic Dynamo Model}

The full-scale nonlinear theory of the astrophysical dynamos is, of course, a formidable challenge and, in practical terms, a degree more complicated than even the problem of fluid turbulence. One must therefore try to find simplified models in order to be able to extract any theoretical understanding of the magnetic dynamo problem. The kinematic stage of the magnetic-field growth, when the field is so weak that it exerts no appreciable back force on the medium, has traditionally been the focus of theoreticians' attention. Indeed, if the velocity field is decoupled from the magnetic field and can be fed into the problem in some given form, the induction equation becomes formally linear (in the magnetic field) and the task of solving it starts to appear within theory's reach. Thus, we consider the magnetic field evolving according to the induction equation (formally in $d$ dimensions):

$$
\partial_{t} B^{i}=-u^{k} B_{, k}^{i}+u_{, k}^{i} B^{k}-u_{, k}^{k} B^{i}+\eta \Delta B^{i},
$$

where $u^{i}(t, \mathbf{x})$ is the externally specified advecting velocity field, $u_{, k}^{i}=\partial u^{i} / \partial x^{k}, B_{, k}^{i}=\partial B^{i} / \partial x^{k}$, and the Einstein summation over repeated indices is used throughout. Two basic avenues of research have been pursued in the literature on the kinematic dynamo problem. One of them is to study the magnetic-field amplification in some prescribed deterministic, though possibly chaotic (i.e. stationary and stochastic in space), fluid flows and/or in specific geometries (see, e.g., Moffatt 1978; Ruzmaikin, Shukurov, and Sokoloff 1988; Childress and Gilbert 1995). Another approach is to consider random (in time) velocity fields with prescribed statistics and try to determine the resulting statistics of the magnetic field (Kazantsev 1968; Kulsrud and Anderson 1992). As the dynamo models of the latter kind appear to better reflect the turbulent nature of the fluid motions that occur in the astrophysical applications of interest to us (ISM and protogalaxies: see $\S 1.1$ ), we restrict ourselves to such statistical line of research only. Additional assumptions of spatial homogeneity and isotropy of the system and statistical stationarity of the velocity field are usually made. Furthermore, we assume mirror-invariance, thus excluding the helicity effects, which are not expected to be felt at the time scales relevant to the small-scale-field physics (see discussion in $\S 1.1$ above and in Kulsrud and Anderson 1992).

Unfortunately, the kinematic assumption alone is not sufficient to turn the turbulent dynamo problem into a tractable one. The realistic turbulent velocity fields possess nontrivial intermittent statistics, which are hard to handle. It is necessary to assume something about them, fully appreciating the fact that any such assumption is bound to be highly artificial. An obvious such assumption, to which many turbulence theories gravitate, is to consider a Gaussian velocity field. This brings about an enormous simplification due to the splitting property of the Gaussian averages. However, even the Gaussian assumption does not fully remove the usual turbulence moment closure problem. The remaining complication has to do with the fact that, in general, the advecting flow is finite-time correlated.

When the advecting velocity field has a finite correlation time, the equations for statistical quantities

\footnotetext{
4 “... I became convinced ... that the best I could do with all the notions that I had thitherto taken into my credence was to have the good sense of ridding my mind thereof, in order to subsequently put back in either other, better ones, or even the same, once I had arranged them according to reason" (Descartes, Discourse on Method).
} 
such as the moments of the magnetic field cannot be obtained in a closed form. Moreover, introducing a finite velocity correlation time into the problem leads to sensitive dependence of the resulting statistics on the specific structure of the velocity correlations in time and space. [For more detailed discussion of these issues and further results with regard to the finite-correlation-time effects, we address the reader to papers by van Kampen (1976); Boldyrev (2000); Schekochihin and Kulsrud (2001), and references therein.] In order to obtain a solvable kinematic dynamo model, we must therefore take another drastic simplifying step. This consists in assuming that the velocity field, besides being Gaussian, also possesses the white-noise property, i.e., it is a random process $\delta$-correlated in time:

$$
\left\langle u^{i}(t, \mathbf{x}) u^{j}\left(t^{\prime}, \mathbf{x}^{\prime}\right)\right\rangle=\delta\left(t-t^{\prime}\right) \kappa^{i j}\left(\mathbf{x}-\mathbf{x}^{\prime}\right) .
$$

Such a synthetic velocity field is sometimes called the Kraichnan ensemble, in deference to the author who first proposed it as a model for studying the passive-scalar problems such as the advection of temperature, or of concentration of an admixture (Kraichnan 1968, 1994). In application to the magnetic fields, this model was proposed independently by Kazantsev (1968), whose work, in fact, predates Kraichnan's. In physical terms, the Kazantsev-Kraichnan assumption amounts to the short-correlation-time approximation and is valid if the advecting velocity field is correlated at times much smaller than the characteristic time of the dynamo action. The latter is of the order of the inverse velocity gradient (the eddy-turnover time of the velocity field).

When the white-noise assumption is put in force, the closure problem resolves itself. Thus, one of the main reasons for the Kazantsev-Kraichnan passive-advection paradigm being so attractive is that it is one of the few available "Ersatz-models" of turbulence, which hold promise of exact solvability. However, the existing theory is not complete, and the statistics of passive advection continue to generate considerable interest, for the problem of passive advection can serve as a vehicle for developing a set of analytical tools appropriate for attacking more realistic turbulence models.

The objective of this paper will be to develop a theory that would allow us to calculate, under the assumptions explained above, the two-point second-order correlation function of the advected field $B^{i}$ :

$$
\left\langle B^{i}(t, \mathbf{x}) B^{j}\left(t, \mathbf{x}^{\prime}\right)\right\rangle=H^{i j}\left(t, \mathbf{x}-\mathbf{x}^{\prime}\right) .
$$

Here and everywhere in this work, the angle brackets denote ensemble averaging with respect to the random advecting velocity field $u^{i}$, as well as with respect to the initial distribution of $B^{i}$. The latter, however, should not greatly affect matters after a finite transient time has elapsed (physically, about one eddyturnover time). The two-point, second-order correlation functions have a clear physical interpretation: their Fourier transforms are the energy spectra.

\subsection{General Scenario of the Small-Scale-Field Dynamo}

The study of the kinematic dynamo problem in the above formulation was pioneered by Kazantsev (1968). Kazantsev reduced the problem of finding the two-point correlation functions of the fluctuating magnetic fields to solving a certain quantum mechanical problem that described a particle with variable (position-dependent) mass moving in a one-dimensional potential well. The eigenfunctions and the energy levels of the particle's Hamiltonian corresponded to the magnetic-field correlation functions with stationary spatial profiles and their overall growth rates, respectively. The ground state of the particle gave rise to the largest growth rate and thus determined the long-time evolution of the magnetic fluctuations. Importantly, 
the exact shape of the potential depended on the particular form of the velocity correlation functions. ${ }^{5}$

In order to extract the general properties of the problem, Kazantsev concentrated his attention on the fluctuating magnetic fields at scales very small compared to the correlation length of the velocity field. In this limit, the two-point velocity correlation functions, which depend on the distance between the points, can be expanded around the origin. Two principal subclasses of the two-point kinematic-dynamo problem can be identified in this context, depending on the physical setting one is interested in. If a problem with a large magnetic Prandtl number is considered, i.e., if the resistive-diffusive scale $k_{\eta}^{-1}$ is much smaller than the characteristic scale $k_{\nu}^{-1}$ of the advecting flow, viz., $k_{\eta} / k_{\nu} \sim \operatorname{Pr}^{1 / 2} \gg 1$, we find ourselves dealing with what is customarily referred to in the literature as the Batchelor regime [after the original work of Batchelor (1959) where this view of passive advection was first elaborated]. In this case, the Taylor expansion of the velocity correlator $\kappa^{i j}(\mathbf{y})$ can be used, whose lowest-order $\mathbf{y}$-dependent term is quadratic (see Appendix A). Since in the astrophysical applications that are of most interest to us (the interstellar medium and the protogalactic plasma), the Prandtl number is very large, it is the Batchelor regime that we shall concentrate on in this work. The models with small Prandtl numbers, where the scale ratio between the flow and the magnetic field is reversed, $k_{\eta} / k_{\nu} \sim \operatorname{Pr}^{1 / 2}<1$, lead to nonanalytic $\mathbf{y}$ dependence for the velocity correlator that magnetic fluctuations "feel" at small scales. Slightly different quantum mechanics result (Kazantsev 1968; Vainshtein 1982a,b; Kichatinov 1985; Vainshtein and Kichatinov 1986; Vergassola 1996; Rogachevskii and Kleeorin 1997). These matters fall beyond the scope of this paper and will be reported elsewhere.

Let us now outline the general scenario that emerges from the investigation of the statistical evolution of small-scale fluctuating magnetic fields in the Batchelor regime (Kulsrud and Anderson 1992). Let us assume that, initially, the magnetic fields are excited at scales comparable to the characteristic scale of the advecting flow $k_{\nu}^{-1}$. (In the astrophysical context discussed above, this assumption is consistent with the current undertanding of the physics of seed fields — see $\S 1.1$ and references therein.) Due to the convective and line-stretching ("dynamo") action of the velocity field, two processes are initiated and proceed exponentially fast in time, at the rates comparable to the eddy-turnover rate of the velocity field. First, the magnetic fluctuations spread over a widening range of scales, their bulk shifting towards ever-smaller ones. A powerlike spectrum forms behind the advancing peak. Second, the level of excitation (amplitude) of each spatial Fourier mode either grows (in 3D) or decreases (in 2D). The combination of these two processes results in the exponential growth of the total magnetic energy. Fig. 1 and Fig. 2 illustrate the evolution of the magnetic-fluctuation spectra in two and three dimensions respectively. Note that in $2 \mathrm{D}$, the overall growth of the energy is due entirely to the spreading of the excitation over an increasing number of modes, while in $3 \mathrm{D}$, each mode also grows by itself. We call this stage of the kinematic dynamo the diffusion-free regime.

Eventually, the magnetic fluctuations reach the diffusive (resistive) scales $\sim k_{\eta}^{-1}$. The effect of the Ohmic dissipation is to check the decrease of the characteristic scale of the magnetic field. The evolution of the magnetic energy is now determined exclusively by the exponential growth (in 3D) or decay (in 2D) of the level of excitation in each particular mode.

We will see in our analysis that, while the evolution of the magnetic fluctuations starts at scales comparable to those of the advecting velocity (henceforth referred to as large, or integral, scales), practically all of their energy will shift to the small scales, $k \gg k_{\nu}$, after a few eddy-turnover times. Thereupon, the spectral

\footnotetext{
${ }^{5}$ Recently it was demonstrated that statistics of general passive tensor fields, such as gradients of temperature or concentration, tensor products of vectors, etc., can also be reduced to a quantum mechanical problem. Remarkably, this problem is exactly solvable and describes $d$ particles on the line which interact pairwise with the potential $\sim 1 /\left(x_{i}-x_{j}\right)^{2}$, the so called Calogero-Sutherland potential (Bernard, Gawędzki, and Kupiainen 1998; Boldyrev and Schekochihin 2000).
} 
properties of the small-scale fields can for the most part be understood in disregard of the small fraction of the magnetic energy that is situated at the large scales. The auxiliary model where the effect of the large scales is neglected and all the magnetic energy is considered to be concentrated at small scales, will also be called the diffusion-free regime before the resistive scales are reached, and the resistive regime afterwards. For both cases, exact Green's-function solutions can be obtained (see $\S 2.3$ and $\S 2.4$ ).

The eventual spreading of the excitation over all scales available in the system, including the resistive and the large, ushers the true long-time asymptotic regime (or the long-time limit), which was the principal subject of Kazantsev's investigation. In three dimensions, a stationary correlation profile is expected to form which, if the Prandtl number exceeds a certain critical value, continues to grow exponentially. This is the eigenstate arising from Kazantsev's quantum mechanics, and Kazantsev's objective was to determine the growth rate and the scaling of the correlation function.

While the analysis of the small-scale asymptotics allowed him to constrain this growth rate between certain, relatively narrow, bounds, Kazantsev identified the main difficulty of the theory: effectively, the potential that appeared in his quantum mechanics was of the inverse-square kind $\left(\sim-1 / x^{2}\right)$ and therefore had to be regularized at both small (resistive) and large (velocity-field) scales, in order for the eigenstates of the problem to be determined (see, e.g., Morse and Feshbach 1953). While on the small-scale side the resistive regularization could be included exactly, the boundary conditions at large scales were unknown, so there was not enough information to fix the dynamo growth rate. A number of authors took up the study of Kazantsev's quantum-mechanical model in the 1980's. Much mathematical insight was gained into the properties of Kazantsev's quantum mechanics, but the integral-scales problem was mostly circumvented by choosing particular reasonable forms of the velocity correlation function (Ruzmaikin and Sokoloff 1981; Novikov, Ruzmaikin, and Sokoloff 1983; Artamonova and Sokoloff 1986; Kleeorin, Ruzmaikin, and Sokoloff 1986; Maslova and Ruzmaikin 1987).

The particular cases analyzed in the literature indicate that the second-order statistics (energy spectra and growth rates) are insensitive to the exact form of the large-scale regularization (see Novikov, Ruzmaikin, and Sokoloff 1983; Artamonova and Sokoloff 1986; Kleeorin, Ruzmaikin, and Sokoloff 1986; Maslova and Ruzmaikin 1987; Kulsrud and Anderson 1992; Subramanian 1997, 1998, 1999, §3.3, and Appendix C below). The growth rate in three dimensions can be determined exactly and turns out to be slightly smaller $\left[\right.$ by $\left.\sim 1 /(\ln \mathrm{Pr})^{2}\right]$ than the growth rate of each individual mode that operated in the diffusion-free regime. In two dimensions, the steadily growing bound eigenstates do not exist, and a continuous spectrum of eigenvalues is formed that is bounded only by zero and therefore indicates that the exponential decay of the magnetic fluctuations in the resistive regime is replaced by a power-like decay in the long-time limit (Novikov, Ruzmaikin, and Sokoloff 1983). In this context, we recall that, as was predicted most generally by Zeldovich's antidynamo theorem (Zeldovich 1957), only transitory growth is possible in two dimensions, and the magnetic field must eventually be dissipated by the resistivity.

The goal of this paper is to give a coherent general exposition of the two-point second-order statistical theory of the kinematic dynamo. The emphasis is naturally placed upon the universal aspects of the problem, which emerge when the statistics of small-scale fields are considered. As long as specific functional features of the velocity correlation function (2) remain inessential, only its small-scale expansion affects the statistics of the passive magnetic fields:

$$
\kappa^{i j}(\mathbf{y})=\kappa_{0} \delta^{i j}-\frac{1}{2} \kappa_{2}\left(y^{2} \delta^{i j}+2 a y^{i} y^{j}\right)+\cdots .
$$

In this case, the kinematic dynamo problem contains only two essential dimensionless parameters: the dimension of space $d$ and the degree of compressibility $a$ of the velocity field. Rather than considering various 
particular cases, such as incompressible, $a=-1 /(d+1)$, irrotational, $a=1$, three-, or two-dimensional dynamo, we work in general, explicitly keeping all dependences on these parameters. This enables us to construct a solid theoretical framework which incorporates all previously available results, elicits their underlying structure, and allows us to establish several previously unknown features and facets of the kinematic dynamo problem. Besides the general evolution equations for the magnetic-field spectra and configurationspace correlation functions that are derived for arbitrary compressibility and spatial dimension [such as equations (11), (21), (56), and (61)], the new results include, for example, the exact Green's-function solution for the small-scale-field spectra in the resistive regime [Eq. (35)] and a simple regularization procedure that allows to calculate the dynamo growth rates in the long-time limit (§3.3).

This paper contains three more Sections and three Appendices. In Sec. 2, the kinematic dynamo problem is studied in its spectral form. Time evolution of the magnetic energy and its propagation over scales are described up to the point when all available scales are excited: from those where the velocity field operates to those at which the magnetic energy is diffused by the Ohmic resistive damping. In Sec. 3, we study the two-point correlation functions of the magnetic fluctuations in the spirit of Kazantsev's configurationspace quantum-mechanical approach, which is convenient for handling the kinematic dynamo problem at all scales in the long-time limit. Each of these sections starts with a brief summary of its internal organization and of the results contained therein. Sec. 4 contains concluding remarks of physical nature. In particular, we discuss the possible directions of future research and some strategies whereby the yet-to-be-developed nonlinear small-scale-dynamo theory could be built upon the foundation laid by the kinematic theory worked out in this paper. Under Appendix A, we include a number of technical appendices dealing with the basic properties of the correlation functions of isotropic fields. In Appendix B, we state the fundamental Gaussian averaging theorem that lies at the core of the derivations of closed equations for the correlation functions in the Kazantsev-Kraichnan model: the Furutsu-Novikov formula (Furutsu 1963; Novikov 1965). Finally, Appendix C contains some overflow asymptotic results pertaining to the material of Sec. 3.

\section{SPECTRA OF MAGNETIC FLUCTUATIONS}

We will start our investigation of the kinematic dynamo by looking at the spectra of magnetic fluctuations. While in most of the works on the subject (Kazantsev 1968; Ruzmaikin and Sokoloff 1981; Molchanov, Ruzmaikin, and Sokoloff 1983; Novikov, Ruzmaikin, and Sokoloff 1983; Kichatinov 1985; Vainshtein and Kichatinov 1986; Artamonova and Sokoloff 1986; Kleeorin, Ruzmaikin, and Sokoloff 1986; Maslova and Ruzmaikin 1987; Vergassola 1996; Rogachevskii and Kleeorin 1997; Subramanian 1997, 1998, 1999), the configuration-space view was chosen because of a transparent quantum-mechanical form the problem took, it is easier to gain a general physical understanding of the processes under way if one first gains a firm foothold in the wave-number space. The statistical-evolution scenario outlined in the Introduction, viz., the growth and spreading of the magnetic excitation over scales, will naturally emerge in the $\mathbf{k}$-space description. Another important consideration is that the correlation functions are better recognized as such in the Fourier space, while in the $\mathbf{x}$ space, only the functions that are Fourier transforms of the proper $\mathbf{k}$-space correlation functions constitute the class of allowed solutions (see Appendix A.1).

The evolution equation for the magnetic-field spectrum was first derived by Kazantsev (1968) and, in a somewhat different, but equivalent, form, by Kraichnan and Nagarajan (1967). Kulsrud and Anderson (1992) later developed a rather detailed theory of magnetic fluctuation spectra. Here we derive some of the

existing results in a more general form (in $d$ dimensions and for arbitrary degree of compressibility of the advecting flow) and then proceed to present several new results on the spectral theory of small-scale fields. 
In $\S 2.1$, we derive the general integral equation that governs the evolution of the magnetic spectrum. From it, we infer the exponential growth of magnetic energy and the exponential decrease of the characteristic scale of magnetic fluctuations. In $\S 2.2$, we obtain a partial differential equation that describes the behavior of the magnetic spectra at scales much smaller than those of the advecting flow. In $\S 2.3$, we study the exact Green's-function solution for the spectrum of the small-scale magnetic fluctuations in the diffusion-free regime. In $\S 2.4$, we proceed to the resistive regime. The previously unknown exact Green's-function solution is obtained in quadratures that is valid simultaneously in both inertial and resistive scale ranges. In $\S 2.5$, we take up the question of the long-time asymptotics of the magnetic spectra that emerge after both resistive and integral scales have been excited. The latter problem is better suited to be treated in the configuration space. This brings us to the material presented in Sec. 3 .

\subsection{The General Equation for the Magnetic-Energy Spectrum}

We shall consider the kinematic dynamo problem as a problem of Gaussian passive advection defined by equations (1) and (2). The velocity correlation function in the wave-number space is, by definition,

$$
\begin{aligned}
\left\langle u^{i}(t, \mathbf{k}) u^{j}\left(t^{\prime}, \mathbf{k}^{\prime}\right)\right\rangle & =\delta\left(t-t^{\prime}\right)(2 \pi)^{d} \delta\left(\mathbf{k}+\mathbf{k}^{\prime}\right) \kappa^{i j}(\mathbf{k}), \\
\kappa^{i j}(\mathbf{k}) & =\kappa(k) \delta^{i j}+\tilde{\kappa}(k) \frac{k_{i} k_{j}}{k^{2}}
\end{aligned}
$$

(in the case of incompressible flow, $\tilde{\kappa}=-\kappa$, while for the purely irrotational flow, $\kappa=0$ ). We will also sometimes use the spectral energy function $I(k)=\kappa^{i i}(k)=d \kappa(k)+\tilde{\kappa}(k)$. The magnetic correlation function is defined as follows:

$$
\begin{aligned}
\left\langle B^{i}(t, \mathbf{k}) B^{j}\left(t, \mathbf{k}^{\prime}\right)\right\rangle & =(2 \pi)^{d} \delta\left(\mathbf{k}+\mathbf{k}^{\prime}\right) H^{i j}(t, \mathbf{k}), \\
H^{i j}(t, \mathbf{k}) & =\frac{1}{d-1} H(t, k)\left(\delta^{i j}-\frac{k_{i} k_{j}}{k^{2}}\right) .
\end{aligned}
$$

The induction equation (1) in the Fourier space can be written as follows (from here on, we suppress the time dependence in the arguments unless it is essential for the clarity of exposition):

$$
\partial_{t} B^{i}(\mathbf{k})+\eta k^{2} B^{i}(\mathbf{k})=i k_{l} \int \frac{\mathrm{d}^{d} k^{\prime}}{(2 \pi)^{d}}\left[u^{i}\left(\mathbf{k}^{\prime}\right) B^{l}\left(\mathbf{k}-\mathbf{k}^{\prime}\right)-u^{l}\left(\mathbf{k}^{\prime}\right) B^{i}\left(\mathbf{k}-\mathbf{k}^{\prime}\right)\right] .
$$

We can now write an equation for the tensor $B^{i}(t, \mathbf{k}) B^{j}\left(t, \mathbf{k}^{\prime}\right)$ and average it:

$$
\begin{gathered}
\partial_{t}\left\langle B^{i}(\mathbf{k}) B^{j}\left(\mathbf{k}^{\prime}\right)\right\rangle+\eta\left(k^{2}+k^{\prime 2}\right)\left\langle B^{i}(\mathbf{k}) B^{j}\left(\mathbf{k}^{\prime}\right)\right\rangle= \\
\int \frac{\mathrm{d}^{d} k^{\prime \prime}}{(2 \pi)^{d}}\left\{i k_{l}\left[\left\langle u^{i}\left(\mathbf{k}^{\prime \prime}\right) B^{l}\left(\mathbf{k}-\mathbf{k}^{\prime \prime}\right) B^{j}\left(\mathbf{k}^{\prime}\right)\right\rangle-\left\langle u^{l}\left(\mathbf{k}^{\prime \prime}\right) B^{i}\left(\mathbf{k}-\mathbf{k}^{\prime \prime}\right) B^{j}\left(\mathbf{k}^{\prime}\right)\right\rangle\right]\right. \\
\left.+i k_{l}^{\prime}\left[\left\langle u^{j}\left(\mathbf{k}^{\prime \prime}\right) B^{l}\left(\mathbf{k}^{\prime}-\mathbf{k}^{\prime \prime}\right) B^{i}(\mathbf{k})\right\rangle-\left\langle u^{l}\left(\mathbf{k}^{\prime \prime}\right) B^{j}\left(\mathbf{k}^{\prime}-\mathbf{k}^{\prime \prime}\right) B^{i}(\mathbf{k})\right\rangle\right]\right\}
\end{gathered}
$$

The mixed triple averages that have arisen in the right-hand side are split by the Furutsu-Novikov (or "Gaussian-integration") formula (see Appendix B) into products of velocity correlation functions defined in the formula (5) and averaged response functions:

$$
\begin{aligned}
\left\langle B^{i}\left(t, \mathbf{k}_{1}\right) B^{j}\left(t, \mathbf{k}_{2}\right) u^{l}\left(t, \mathbf{k}_{3}\right)\right\rangle & =\int^{t} \mathrm{~d} t^{\prime} \int \mathrm{d}^{d} k^{\prime}\left\langle u^{l}\left(t, \mathbf{k}_{3}\right) u^{n}\left(t^{\prime}, \mathbf{k}^{\prime}\right)\right\rangle\left\langle\frac{\delta\left[B^{i}\left(t, \mathbf{k}_{1}\right) B^{j}\left(t, \mathbf{k}_{2}\right)\right]}{\delta u^{n}\left(t^{\prime}, \mathbf{k}^{\prime}\right)}\right\rangle \\
& =\frac{1}{2}(2 \pi)^{d} \kappa^{l n}\left(\mathbf{k}_{3}\right)\left\langle\frac{\delta\left[B^{i}\left(t, \mathbf{k}_{1}\right) B^{j}\left(t, \mathbf{k}_{2}\right)\right]}{\delta u^{n}\left(t,-\mathbf{k}_{3}\right)}\right\rangle .
\end{aligned}
$$


The averaged same-time response function that appears above is

$$
\begin{gathered}
\left\langle\frac{\delta\left[B^{i}\left(\mathbf{k}_{1}\right) B^{j}\left(\mathbf{k}_{2}\right)\right]}{\delta u^{n}\left(-\mathbf{k}_{3}\right)}\right\rangle=i k_{1 m}\left[\delta^{i n} H^{m j}\left(\mathbf{k}_{1}+\mathbf{k}_{3}\right)-\delta^{m n} H^{i j}\left(\mathbf{k}_{1}+\mathbf{k}_{3}\right)\right] \delta\left(\mathbf{k}_{1}+\mathbf{k}_{2}+\mathbf{k}_{3}\right) \\
+i k_{2 m}\left[\delta^{j n} H^{m i}\left(\mathbf{k}_{2}+\mathbf{k}_{3}\right)-\delta^{m n} H^{i j}\left(\mathbf{k}_{2}+\mathbf{k}_{3}\right)\right] \delta\left(\mathbf{k}_{1}+\mathbf{k}_{2}+\mathbf{k}_{3}\right),
\end{gathered}
$$

where the tensor $H^{i j}(\mathbf{k})$ is defined by the formula (6). The expression (10) is obtained by formally integrating the (unaveraged) equation for $B^{i}\left(t, \mathbf{k}_{1}\right) B^{j}\left(t, \mathbf{k}_{2}\right)$ up to time $t$, taking the functional derivative $\delta / \delta u^{n}\left(t^{\prime}, \mathbf{k}_{3}\right)$, averaging, setting $t^{\prime}=t$, and using the causal property of the response functionals.

Using the definitions (5) and (6) and after some straightforward algebra, we arrive at the so called mode-coupling equation:

$$
\begin{gathered}
\partial_{t} H+2\left(\eta_{T}+\eta\right) k^{2} H= \\
\frac{1}{d-1} \int \frac{\mathrm{d}^{d} k^{\prime \prime}}{(2 \pi)^{d}}\left\{(d-1) k^{2} \kappa\left(k^{\prime \prime}\right)+\frac{2\left(\mathbf{k} \cdot \mathbf{k}^{\prime}\right)\left(\mathbf{k} \cdot \mathbf{k}^{\prime \prime}\right)\left(\mathbf{k}^{\prime} \cdot \mathbf{k}^{\prime \prime}\right)}{k^{\prime 2} k^{\prime \prime}} \tilde{\kappa}\left(k^{\prime \prime}\right)\right. \\
+\left[k^{2}-\frac{\left(\mathbf{k} \cdot \mathbf{k}^{\prime}\right)^{2}}{k^{\prime 2}}\right]\left[\kappa\left(k^{\prime \prime}\right)+\tilde{\kappa}\left(k^{\prime \prime}\right)\right] \\
\left.+(d-3)\left[k^{2}-\frac{\left(\mathbf{k} \cdot \mathbf{k}^{\prime}\right)^{2}}{k^{\prime 2}}\right] \kappa\left(k^{\prime \prime}\right)+(d-3) \frac{\left(\mathbf{k} \cdot \mathbf{k}^{\prime \prime}\right)^{2}}{k^{\prime \prime 2}} \tilde{\kappa}\left(k^{\prime \prime}\right)\right\} H\left(k^{\prime}\right),
\end{gathered}
$$

where $\mathbf{k}^{\prime}=\mathbf{k}-\mathbf{k}^{\prime \prime}$, and we have introduced an auxiliary quantity that one may term the turbulent diffusivity:

$$
\eta_{T}=\frac{1}{d} \int \frac{\mathrm{d}^{d} k}{(2 \pi)^{d}} I(k)
$$

[recall that $I(k)=d \kappa(k)+\tilde{\kappa}(k)$ ]. Eq. (11) is the generalization of the mode-coupling equations of Kazantsev (1968), Vainshtein (1982a), and of Kulsrud and Anderson (1992) to the case of $d$ dimensions and arbitrary degree of compressibility. It is now straightforward to verify that Eq. (11) integrated over all wave numbers gives the following evolution law of the total magnetic energy $W=(2 \pi)^{-d} \int \mathrm{d}^{d} k H(k)$ :

$$
\partial_{t} W=2 \gamma W-2 \eta \overline{k^{2}} W
$$

where the growth rate is

$$
2 \gamma=\frac{d-1}{d} \int \frac{\mathrm{d}^{d} k}{(2 \pi)^{d}} k^{2}[2 \kappa(k)+\tilde{\kappa}(k)]
$$

and the mean square wave number of the magnetic spectrum is

$$
\overline{k^{2}}=\frac{1}{W} \int \frac{\mathrm{d}^{d} k}{(2 \pi)^{d}} k^{2} H(k) .
$$

The non-conservation of the magnetic energy reflected by Eq. (13) is understandable, because the system is not closed and the passive magnetic field constantly receives energy from the velocity field. Note that the white-in-time velocity field formally constitutes an infinite reservoir of energy.

It is instructive to have also an evolution equation for $\overline{k^{2}}$. This is derived in quite the same fashion as Eq. (13) and reads

$$
\partial_{t} \overline{k^{2}}=D+2 \gamma_{2} \overline{k^{2}}-2 \eta\left[\overline{k^{4}}-\left(\overline{k^{2}}\right)^{2}\right]
$$


where the growth rate is

$$
2 \gamma_{2}=\frac{1}{d} \int \frac{\mathrm{d}^{d} k}{(2 \pi)^{d}} k^{2}\left[I(k)+4\left(\kappa(k)+\frac{d+1}{d+2} \tilde{\kappa}(k)\right)\right],
$$

the source term, which corresponds to a slow diffusion of the magnetic spectrum in $k$ space, is

$$
D=\frac{d-1}{d} \int \frac{\mathrm{d}^{d} k}{(2 \pi)^{d}} k^{4}[2 \kappa(k)+\tilde{\kappa}(k)]
$$

and the "fourth moment" of the magnetic spectrum is defined analogously to the second (15):

$$
\overline{k^{4}}=\frac{1}{W} \int \frac{\mathrm{d}^{d} k}{(2 \pi)^{d}} k^{4} H(k) .
$$

All of the velocity-spectrum moments introduced above [formulas (14), (17), and (18)] can be shown to be positive based on the realizability properties of the isotropic velocity-field correlation functions (see Appendix A.3). Note that, since $\overline{k^{4}}-\left(\overline{k^{2}}\right)^{2}=\overline{\left(k^{2}-\overline{k^{2}}\right)^{2}} \geq 0$, the resistive term still plays the ultimately balancing role in Eq. (16).

Eq. (13) and Eq. (16) provide us with a basic understanding of the time-evolution properties of the magnetic energy spectrum. Let us assume that initially the magnetic fluctuations are concentrated at wave numbers comparable with or greater than the characteristic wave number $k_{\nu}$ of the velocity field, and that the Prandtl number is large, i.e., the characteristic wave number associated with Ohmic resistive diffusion, $k_{\eta} \sim \operatorname{Pr}^{1 / 2} k_{\nu}$, greatly exceeds $k_{\nu}$. We see that the mean square wave number of the magnetic fluctuations increases exponentially in time with the growth rate $\gamma_{2}$, which is of the same order as that of the magnetic energy, until this increase is checked by the resistive dissipation [Eq. (16)]. Thus, the bulk of the magnetic spectrum will shift towards the resistive scale range exponentially fast with characteristic rate $\gamma_{2}$. The fluctuations themselves will also increase in strength, exponentially fast as well, with the rate $\gamma$ [Eq. (13)].

Naturally, the question arises, what is going to happen when the resistive scales are finally reached. That is where the principal technical difficulties of this theory lie. Before we attack this problem, let us probe a little further into the additional information Eq. (11) can yield regarding the properties of the spectra of small-scale fields.

\subsection{The Small-Scale-Field Equation}

We saw in the previous section that the spectrum of magnetic fluctuations had a tendency to shift to scales much smaller than those of the velocity field. This allows us to study the magnetic energy spectra in what we shall call the small-scale approximation. Namely, we may notice that the kernel of the integral operator in the right-hand side of the mode-coupling equation (11) essentially cuts off the range of values that $k^{\prime \prime}$ may take at the scales $k^{\prime \prime} \sim k_{\nu}$, and hence, for $k \gg k_{\nu}$, we may expand

$$
\begin{aligned}
H\left(k^{\prime}\right)=H(k) & +\left[\frac{1}{2}\left(\frac{k^{\prime \prime}}{k}\right)^{2} \sin ^{2} \theta-\frac{k^{\prime \prime}}{k} \cos \theta\right] k H^{\prime}(k) \\
& +\frac{1}{2}\left(\frac{k^{\prime \prime}}{k}\right)^{2} \cos ^{2} \theta k^{2} H^{\prime \prime}(k)+\mathcal{O}\left[\left(k^{\prime \prime} / k\right)^{3}\right],
\end{aligned}
$$

where $\theta$ is the angle between $\mathbf{k}^{\prime \prime}$ and $\mathbf{k}$. Expanding similarly the kernel and performing the necessary angle integrations (with the aid of Appendix A.5), we arrive at the following small-scale-field (SSF) equation:

$$
\partial_{t} H+2 \eta k^{2} H=\gamma\left(A k^{2} H^{\prime \prime}+B k H^{\prime}+C H\right),
$$


where $\gamma$ is defined by the formula (14), while the dimensionless coefficients $A, B$, and $C$ depend only on the dimension of space and the degree of compressibility of the velocity field:

$$
\begin{aligned}
A & =\frac{1+2 a}{(d-1)(d a+2)}, \\
B & =\frac{d-1+4 a}{(d-1)(d a+2)}, \\
C & =\frac{2(d-2)}{(d-1)(d a+2)} .
\end{aligned}
$$

Here $a$ is the so-called compressibility parameter of the velocity field which arises naturally the smallscale expansion of the velocity correlation function and is ubiquitous in one-point statistical theories (see Appendix A.4 and Boldyrev and Schekochihin 2000; Schekochihin and Kulsrud 2001):

$$
a=\left[1+(d+2) \int \frac{\mathrm{d}^{d} k}{(2 \pi)^{d}} k^{2} \kappa(k) / \int \frac{\mathrm{d}^{d} k}{(2 \pi)^{d}} k^{2} \tilde{\kappa}(k)\right]^{-1} .
$$

By using the realizability properties of the velocity correlation functions (see Appendix A.3), we find that the values of $a$ are sandwiched between $-1 /(d+1)$ (incompressible flow) and unity (irrotational flow), $A>0$, the sign of $B$ is undetermined, and $C>0$ except in $2 \mathrm{D}$, where it vanishes. The values of $A, B$, and $C$ in the four special cases that are usually studied in the literature are given in Table 2. In the case of incompressible flow, Eq. (21) was earlier obtained in 3D by Kazantsev (1968) (see also Vainshtein 1980, 1982b; Kulsrud and Anderson 1992) and in $d$ dimensions by Gruzinov, Cowley, and Sudan (1996).

It is worthwhile noticing that $A(d+1) d+B d+C=2$, and therefore the SSF equation (21) satisfies the exact energy evolution law (13). Thus, the small-scale approximation is conservative. This is an essential property because it means that all of the magnetic energy is concentrated at the small scales. It is therefore reasonable to expect that the spectral properties of the small-scale fields would mostly be captured by the SSF equation (21). This consideration encourages us to embark on a detailed investigation of the solutions of this equation.

\subsection{Spreading of Magnetic Fluctuations in the Wave-Number Space: Diffusion-Free Regime}

First, let us pick a wave number $k_{0}$ such that $k_{\nu} \ll k_{0} \ll k_{\eta}$, and inquire how an initial spectrum concentrated at that wave number will evolve over time. This is easily determined, because neglecting the diffusive term in Eq. (21) and changing to the logarithmic variable $z=\ln k$, renders this equation a onedimensional heat equation in a moving frame. The Green's function for such an equation is well known, so we find that an initial spectrum such that $H_{0}(k) \propto \delta\left(k-k_{0}\right)$ spreads out over time into a widening lognormal profile:

$$
\begin{aligned}
M(t, k) & =\frac{S_{d}}{(2 \pi)^{d}} k^{d-1} H(t, k) \\
& =\frac{W_{0}}{k_{0}} e^{\lambda_{0} \gamma t}\left(\frac{k}{k_{0}}\right)^{\xi_{0}} \frac{1}{\sqrt{4 \pi A \gamma t}} \exp \left\{-\frac{\left[\ln \left(k / k_{0}\right)\right]^{2}}{4 A \gamma t}\right\},
\end{aligned}
$$


where $S_{d}=2 \pi^{d / 2} / \Gamma(d / 2)$ is the area of the unit sphere in $d$ dimensions, $W_{0}$ is the initial magnetic energy, and we have introduced two new parameters:

$$
\begin{aligned}
\xi_{0} & =d-1+s_{0}, \quad \text { where } \quad s_{0}=\frac{A-B}{2 A}=\frac{2-d-2 a}{2(1+2 a)}, \\
\lambda_{0} & =C-A s_{0}^{2}=\frac{(3-d)(d-1+4 a)+4(d-2)(1+2 a)-(1+2 a)^{2}}{4(d-1)(1+2 a)(d a+2)} \\
& =2-A\left(\xi_{0}+1\right)^{2} .
\end{aligned}
$$

The values of $s_{0}, \xi_{0}$, and $\lambda_{0}$ in the standard cases are given in Table 2 .

Examining the solution (26), we immediately conclude that the following basic processes are initiated: (i) the fluctuation strength in each logarithmic wave-number band grows or decays exponentially at the rate $\lambda_{0} \gamma$, (ii) the number of such bands that are excited [i.e., the width of the lognormal envelope in the solution (26)] grows exponentially fast at the rate $4 A \gamma$, (iii) a magnetic spectrum with exponent $\xi_{0}$ is formed inside the lognormal envelope, with the peak of the spectrum moving rightwards (to smaller scales/larger $k$ ): $k_{\text {peak }}=k_{0} \exp \left(2 \xi_{0} A \gamma t\right)$. It is not hard to verify that the effective sum of these processes produces the total magnetic-energy growth rate we have previously obtained [Eq. (13)]: $2 \gamma=\lambda_{0} \gamma+A\left(\xi_{0}+1\right)^{2} \gamma$.

Let us make several observations regarding the behavior of the parameters of the solution (26) defined by formulas (27) and (28): the spectral exponent $s_{0}$ (or $\xi_{0}$ ), and the fraction $\lambda_{0}$ of the total growth rate that corresponds to the growth or decay of each individual Fourier mode. (The latter is plotted in Fig. 3 as a function of the compressibility parameter $a$ in two and three dimensions.)

(i) In $3 \mathrm{D}$, the spectral slope that forms behind the advancing peak of the spectrum does not depend on the degree of compressibility: $\xi_{0}=3 / 2\left(s_{0}=-1 / 2\right)$ regardless of the value of $a$.

(ii) While the number of excited modes increases exponentially fast regardless of the particular parameter values, individual modes grow in 3D $\left(\lambda_{0}>0\right.$ for all $\left.a\right)$ and decay in 2D $\left(\lambda_{0}<0\right.$ for all $\left.a \neq 0\right)$.

(iii) In $2 \mathrm{D}$ and for $a=0, \lambda_{0}=0$ and $s_{0}=0$. Curiously, this suggests the forming of an equilibrium-like, equipartion spectrum $\left(\xi_{0}=d-1\right)$.

\subsection{Spreading of Magnetic Fluctuations in the Wave-Number Space: Resistive Regime}

The solution (26) will cease to be valid when the magnetic excitation that has resulted from it reaches the integral scales $\sim k_{\nu}$ and/or the resistive scales $\sim k_{\eta}$. Let us first consider the simpler of these two aspects of the problem, namely that where the resistive scales are reached before the integral ones. In this case, the further behavior of the magnetic fluctuations will be determined by the SSF equation (21) with the resistive term retained.

When the diffusive term is allowed to reappear in the SSF equation (21), a Green's function solution can still be obtained in quadratures. In order to accomplish that, we seek the solution in the form

$$
H(t, k)=e^{\lambda_{0} \gamma t} k^{s_{0}} f\left(t, k / k_{\eta}\right),
$$

where $\lambda_{0}$ and $s_{0}$ are defined by the formulas (28) and (27), and $k_{\eta}=(\gamma A / 2 \eta)^{1 / 2}$ is the characteristic wave number of the resistive dissipation. It is then elementary to see that $f(t, x)$ satisfies

$$
\partial_{t} f=A \gamma\left(x^{2} f^{\prime \prime}+x f^{\prime}-x^{2} f\right) .
$$


The Green's function solution of this problem can be found by applying to Eq. (30) the Kontorovich-Lebedev transform (see, e.g., Erdélyi 1954), which, in the form adapted to our problem, can be defined as follows:

$$
\begin{aligned}
& F(\tau)=\int_{0}^{\infty} \frac{\mathrm{d} x}{x} K_{i \tau}(x) f(x) \quad \text { (direct), } \\
& f(x)=\frac{2}{\pi^{2}} \int_{0}^{\infty} \mathrm{d} \tau \tau \sinh (\pi \tau) K_{i \tau}(x) F(\tau) \quad \text { (inverse) }
\end{aligned}
$$

where $K_{i \tau}(x)$ is the modified Bessel function of the second kind (also known as the Macdonald, or Basset, function). Kontorovich-Lebedev transforming Eq. (30), we get

$$
\partial_{t} F(t, \tau)=-A \gamma \tau^{2} F(t, \tau)
$$

The solution of Eq. (30) is therefore

$$
f(t, x)=\frac{2}{\pi^{2}} \int_{0}^{\infty} \frac{\mathrm{d} x^{\prime}}{x^{\prime}} f_{0}\left(x^{\prime}\right) \int_{0}^{\infty} \mathrm{d} \tau \tau \sinh (\pi \tau) K_{i \tau}(x) K_{i \tau}\left(x^{\prime}\right) e^{-A \gamma \tau^{2} t},
$$

where $f_{0}(x)$ is the initial profile. To compare this result with the inertial-range solution (26), let us recast it in the form of an evolution law for a spectrum that is initially a $\delta$-like spike, $M_{0}(t, k) \propto \delta\left(k-k_{0}\right)$. We have

$$
M(t, k)=\frac{W_{0}}{k_{0}} e^{\lambda_{0} \gamma t}\left(\frac{k}{k_{0}}\right)^{\xi_{0}} \frac{2}{\pi^{2}} \int_{0}^{\infty} \mathrm{d} \tau \tau \sinh (\pi \tau) K_{i \tau}\left(\frac{k}{k_{\eta}}\right) K_{i \tau}\left(\frac{k_{0}}{k_{\eta}}\right) e^{-A \gamma \tau^{2} t} .
$$

It is not hard to verify that, for $k_{0} \ll k_{\eta}$ and $k \ll k_{\eta}$, the solution (26) is recovered as an asymptotic of the more general solution (35). On the opposite end of the spectrum, $k \gg k_{\eta}$, one finds the expected exponential cutoff due to resistive dissipation. Fig. 4 illustrates the evolution of the spectrum (35) with time.

Integrating the spectrum (35), we find that the total magnetic energy evolves according to

$$
\begin{aligned}
W(t)= & W_{0} e^{\lambda_{0} \gamma t}\left(\frac{k_{0}}{k_{\eta}}\right)^{-\left(\xi_{0}+1\right)} \times \\
& \times \frac{2^{\xi_{0}}}{\pi^{2}} \int_{0}^{\infty} \mathrm{d} \tau \tau \sinh (\pi \tau)\left|\Gamma\left(\frac{\xi_{0}+1+i \tau}{2}\right)\right|^{2} K_{i \tau}\left(\frac{k_{0}}{k_{\eta}}\right) e^{-A \gamma \tau^{2} t} .
\end{aligned}
$$

This formula reflects the gradual slide of the "effective growth rate" of the magnetic energy, $\gamma_{\text {eff }}(t)=$ $t^{-1} \ln \left[W(t) / W_{0}\right]$, from the diffusion-free limit $\gamma_{\text {eff }}(t \rightarrow 0)=2 \gamma$ to the diffusion-reduced value, $\gamma_{\text {eff }}(t \rightarrow$ $\infty)=\lambda_{0} \gamma$. This is demonstrated in Fig. 5 .

Direct integration of the solution (35) also produces an exact formula for $\overline{k^{2}}(t)$. Its asymptotic behavior can, however, be inferred already from that of the energy:

$$
\overline{k^{2}}(t \rightarrow \infty)=\frac{2-\lambda_{0}}{A} k_{\eta}^{2}=\left(\xi_{0}+1\right)^{2} k_{\eta}^{2}
$$

[see formula (28)]. This is a good quantitative measure of the characteristic wave number in the resistive range around which the bulk of the magnetic fluctuation energy will stabilize. The evolution of $\left[\overline{k^{2}}(t)\right]^{1 / 2}$ is plotted in Fig. 6.

Thus, the solution (35) describes how the spreading lognormal spectrum hits the resistive scale range and and how any further refinement of the magnetic-fluctuation scales is suppressed by resistive dissipation. The total magnetic energy now concentrates in the resistive scale range and grows (in 3D) or decays (in 2D) at the rate $\lambda_{0} \gamma$. This regime persists until the excitation finally reaches the integral scales. 


\subsection{Spectra and Growth Rates of Magnetic Fluctuations in the Long-Time Limit}

In order to gain some idea of the long-time behavior of the magnetic spectra, which sets in when all scales available in the system, including the integral ones, are excited, let us seek the solution of Eq. (21) in the form

$$
H(t, k)=e^{\lambda \gamma t} h(k)
$$

The constant spectral profile $h(k)$ must then satisfy the following linear ordinary differential equation of the Bessel kind:

$$
A k^{2} h^{\prime \prime}+B k h^{\prime}+\left(C-\lambda-\frac{2 \eta}{\gamma} k^{2}\right) h=0 .
$$

Before considering the explicit solutions of this equation, it is instructive to recast it in a form mathematically equivalent to that of a Schrödinger equation for a particle with energy equal to $-1 / k_{\eta}^{2}$ moving in a onedimensional inverse-square potential, viz.,

$$
-\psi^{\prime \prime}-\left[\frac{1}{4}-\frac{\lambda-\lambda_{0}}{A}\right] \frac{1}{k^{2}} \psi=-\frac{1}{k_{\eta}^{2}} \psi,
$$

where the "wave function" is

$$
\psi(k)=k^{-s_{0}+1 / 2} h(k),
$$

$s_{0}$ and $\lambda_{0}$ are defined by the formulas (27) and (28), and $k_{\eta}=(\gamma A / 2 \eta)^{1 / 2}$ is the inverse resistive scale. The potential in Eq. (40) is repelling and therefore cannot have a negative energy level for $\lambda \geq \lambda_{0}+A / 4$. Solutions of the form (38) do not exist in this case. For $\lambda<\lambda_{0}$, the so-called "fall on center" occurs (see, e.g., Landau and Lifschitz 1977), whereby the solution becomes oscillatory with infinitely many nodes as $k$ approaches zero, so it cannot be a spectral function: for $k \ll k_{\eta}$,

$$
\psi \propto\left(\frac{k}{k_{\eta}}\right)^{1 / 2} \sin \left(\sqrt{\frac{\left|\lambda-\lambda_{0}\right|}{A}} \ln \frac{k}{k_{\eta}}+\text { const }\right) .
$$

Of course, the small-scale approximation is only valid for $k \gg k_{\nu} \sim k_{\eta} \operatorname{Pr}^{-1 / 2}$, so for $\lambda$ lying below $\lambda_{0}$, but within some $\delta \lambda \sim \mathcal{O}\left(1 / \ln ^{2} \operatorname{Pr}\right)$ of it, the solution will not yet have any nodes in the range of wave numbers for which Eq. (40) is valid. Thus, the growth rate of the stationary-profile solution (38) is contained in the interval

$$
\lambda_{0}-\delta \lambda<\lambda<\lambda_{0}+A / 4=\lambda_{\max }, \quad \text { where } \quad \delta \lambda \sim \mathcal{O}\left(\frac{1}{\ln ^{2} \mathrm{Pr}}\right)
$$

For the 3D incompressible case, the bounds (43) on the growth rate of a stationary-profile spectrum, as well as the solution (44), were first established by Kazantsev (1968). He also pointed to the fundamental problem that, since the boundary conditions at small $k$ (integral scales) are unknown, there is no rigorous way to fix the value of $\lambda$ within the framework of the small-scale approximation.

The two fundamental solutions of Eq. (39) are the modified Bessel functions $I_{\nu(\lambda)}\left(k / k_{\eta}\right)$ and $K_{\nu(\lambda)}\left(k / k_{\eta}\right)$ times $k^{s_{0}}$, where $\nu(\lambda)=\sqrt{\left(\lambda-\lambda_{0}\right) / A}$. The obvious requirement that the physically acceptable solution must 
decay at large $k$, leaves us with

$$
\begin{gathered}
M(t, k)=\frac{S_{d}}{(2 \pi)^{d}} k^{d-1} H(t, k)=C(\lambda) e^{\lambda \gamma t}\left(\frac{k}{k_{\eta}}\right)^{\xi_{0}} K_{\nu(\lambda)}\left(\frac{k}{k_{\eta}}\right), \\
C(\lambda)=\frac{W_{0}}{k_{\eta}}\left[2^{\xi_{0}-1} \Gamma\left(\frac{\xi_{0}+1+\nu(\lambda)}{2}\right) \Gamma\left(\frac{\xi_{0}+1-\nu(\lambda)}{2}\right)\right]^{-1},
\end{gathered}
$$

where $K_{\nu}$ is the Macdonald function. The "subresistive" asymptotic of this solution does not depend on $\lambda$ in any essential way and simply reflects the expected exponential cutoff of the magnetic spectrum:

$$
M(t, k \rightarrow \infty) \simeq \sqrt{\frac{\pi}{2}} C(\lambda) e^{\lambda \gamma t}\left(\frac{k}{k_{\eta}}\right)^{\xi_{0}-1 / 2} \exp \left(-\frac{k}{k_{\eta}}\right) .
$$

The asymptotics valid for $k \ll k_{\eta}$ are

$$
\begin{aligned}
& M(t, k) \simeq 2^{\nu(\lambda)-1} \Gamma(\nu(\lambda)) C(\lambda) e^{\lambda \gamma t}\left(\frac{k}{k_{\eta}}\right)^{\xi_{0}-\nu(\lambda)}, \quad \lambda>\lambda_{0}, \\
& M(t, k) \simeq C(\lambda) e^{\lambda_{0} \gamma t}\left(\frac{k}{k_{\eta}}\right)^{\xi_{0}} \ln \left(\frac{k_{\eta}}{k}\right), \quad \lambda=\lambda_{0},
\end{aligned}
$$

and, for $\lambda=\lambda_{0}-\delta \lambda$, where $0<\delta \lambda \ll 1$, we have

$$
M(t, k) \simeq \sqrt{\frac{\delta \lambda}{A}} C(\lambda) e^{\lambda \gamma t}\left(\frac{k}{k_{\eta}}\right)^{\xi_{0}} \sin \left(\sqrt{\frac{\delta \lambda}{A}} \ln \frac{k}{2 k_{\eta}}\right),
$$

which is, of course, consistent with (42).

Clearly, if the initial distribution of the magnetic fluctuations is chosen in such a way that it spreads over to resistive scales before it does to the integral ones (i.e., if the initial characteristic wave number $k_{0}$ in the solution (26) of $\S 2.3$ lies "closer" to $k_{\eta}$ than to $k_{\nu}$ ), there will be an intermediate period between the time the resistive scales are reached and the time when the excitation finally arrives at $k \sim k_{\nu}$. During this period, the boundary condition at the integral scales cannot, of course, affect the spectrum. The solution of the form (38) consistent with the spectra obtained in $\S 2.3$ and $\S 2.4$ [solutions (26) and (35)] will clearly be the one with $\lambda=\lambda_{0}$ : indeed, it can be checked that the solution (44) with $\lambda=\lambda_{0}$ is the long-time asymptotic of the solution (35). This is essentially what was demonstrated numerically by Kulsrud and Anderson (1992) (see also the recent work of Chertkov et al. 1999). Our Green's-function solution (35) is the mathematical expression of this fact.

We may further argue that, if we were to mandate that the solution (44) vanish at some $k_{0}$ such that $k_{\nu} \ll k_{0} \ll k_{\eta}$, then, by virtue of the asymptotic expression (48), we would be required to set

$$
\lambda=\lambda_{0}-\frac{A \pi^{2}}{\left[\ln \left(k_{0} / 2 k_{\eta}\right)\right]^{2}} \simeq \lambda_{0}-\frac{A \pi^{2}}{\left[\ln \left(\operatorname{Pr}^{1 / 2}\right)\right]^{2}} .
$$

Note that the particular value of $k_{0}$ is unimportant here, because $\ln \left(k_{0} / 2 k_{\eta}\right)=\ln \left(k_{\nu} / k_{\eta}\right)+\ln \left(k_{0} / 2 k_{\nu}\right)$, the second term being subdominant. The above value of $\lambda$ specifies a particular solution of the form (38) which exactly vanishes at $k=k_{0}$. In $\S 2.3$ and $\S 2.4$, we saw however that any excitation present at $k>k_{0}$ at a given time must necessarily end up spreading over to the integral scales. The question remains whether, once this happens, the entire spectrum would eventually be affected. It is, however, encouraging to notice that $\lambda=\lambda_{0}$ represents a nontrivial limit when the infrared cutoff $k_{0}$ is taken to zero. 


\section{TWO-POINT CORRELATION FUNCTIONS OF THE MAGNETIC FIELD IN THE CONFIGURATION SPACE}

We would now like to put the solution of the kinematic-dynamo problem in a somewhat different perspective and develop an adequate theory valid in the long-time limit. In order to make any progress, we necessarily have to renounce the small-scale approximation. While it has furnished us with a fair amount of practical understanding of the initial behavior of the magnetic fluctuations in the kinematic regime, it does not contain sufficient information to uniquely fix the growth rates and the spectra eventually attained by the magnetic energy spectrum. We must therefore attack the kinematic dynamo problem in the form that remains valid at all scales. This can be more conveniently accomplished in the configuration space, for the $\mathbf{x}$-space analog (inverse Fourier transform) of the mode-coupling equation (11) is local and can be reduced to the quantum mechanics of a particle with variable mass in a one-dimensional potential well. This approach, first proposed by Kazantsev (1968), leads to a clearer mathematical and physical picture of the properties of the magnetic-field correlation functions in the long-time limit.

Kazantsev's quantum mechanics was studied in the case of 3D incompressible flow by several authors in 1980s (Ruzmaikin and Sokoloff 1981; Novikov, Ruzmaikin, and Sokoloff 1983; Artamonova and Sokoloff 1986; Kleeorin, Ruzmaikin, and Sokoloff 1986; Maslova and Ruzmaikin 1987), mostly for particular forms of the velocity correlation function. Novikov, Ruzmaikin, and Sokoloff (1983) also set forth several important ideas with regard to the $2 \mathrm{D}$ case. In this Section, we develop the configuration-space two-point kinematic dynamo theory for a general, $d$-dimensional and arbitrarily compressible advecting flow. In $\S 3.1$, we derive an evolution equation for the correlation function of the magnetic field. This equation is the configuration-space analog of the mode-coupling equation of the previous Section. We then proceed to formulate the Kazantsev quantum-mechanical form of this equation. In $\S 3.2$, the three principal asymptotic regimes of this model are considered: the asymptotic solutions are found in the subresistive, inertial, and integral scale ranges. In $\S 3.3$, these solutions are matched in a systematic way and the dynamo growth rates are determined for a particular choice of small- and large-scale regularization of the Kazantsev quantum mechanics. The ensuing results have universal applicability if the specific form of the regularization is unimportant. The possible relevance of the large-scale structure of the velocity correlations is further discussed in $\S 3.4$.

\subsection{The Kazantsev Equation}

We start with the induction equation in its standard configuration-space form (1). The usual assumptions of homogeneity, isotropy, and mirror invariance, as well as the Gaussian white-noise nature of the velocity field are made [Eq. (2)]. The velocity correlation tensor in the $\mathbf{x}$ space can be represented as follows:

$$
\kappa^{i j}(\mathbf{y})=\kappa_{L L}(y) \delta^{i j}-\left(\kappa_{L L}(y)-\frac{\kappa_{N N}(y)}{d-1}\right)\left(\delta^{i j}-\frac{y^{i} y^{j}}{y^{2}}\right)
$$

where $\kappa_{L L}(y)$ and $\kappa_{N N}(y)$ are called the longitudinal and the transverse correlation functions. The tensor $\kappa^{i j}(\mathbf{x})$ defined above is the Fourier transform of the $\mathbf{k}$-space correlation tensor $\kappa^{i j}(\mathbf{k})$ whith which we operated in the preceding Section [see Eq. (5)]. The exact relationship between the functions $\kappa_{L L}(y), \kappa_{N N}(y)$ and the $\mathbf{k}$-space correlation functions $\kappa(k), \tilde{\kappa}(k)$ is worked out in detail in Appendix A.1.

We now proceed to derive an evolution equation for the correlation tensor $H^{i j}(t, \mathbf{y})$ of the magnetic field [see definition (3)] by directly averaging the dynamic equation for the tensor $B^{i}(t, \mathbf{x}) B^{j}\left(t, \mathbf{x}^{\prime}\right.$ ) (here, as always, $\mathbf{x}-\mathbf{x}^{\prime}=\mathbf{y}$ ) and splitting the arising triple averages according to the Furutsu-Novikov formula (see 
Appendix B). Namely,

$$
\partial_{t} H^{i j}(\mathbf{y})-2 \eta \Delta H^{i j}(\mathbf{y})=\frac{\partial}{\partial y^{k}}\left[C^{i k j}(\mathbf{y})-C^{k i j}(\mathbf{y})-C^{j k i}(-\mathbf{y})+C^{k j i}(-\mathbf{y})\right],
$$

where the four terms on the right-hand side are the triple averages:

$$
\begin{aligned}
C^{k i j}(\mathbf{y}) & =C^{k i j}\left(\mathbf{x}_{1}-\mathbf{x}_{2}\right)=\left\langle u^{k}\left(\mathbf{x}_{1}\right) B^{i}\left(\mathbf{x}_{1}\right) B^{j}\left(\mathbf{x}_{2}\right)\right\rangle \\
& =\frac{1}{2}\left[\left(\kappa^{k l}(\mathbf{y})-\kappa^{k l}(0)\right) H_{, l}^{i j}(\mathbf{y})-\kappa_{, l}^{k j}(\mathbf{y}) H^{i l}(\mathbf{y})+\kappa_{, l}^{k l} H^{k j}(\mathbf{y})\right] .
\end{aligned}
$$

The details of the derivation of the above expression are standard and straightforward and have therefore been omitted. The reader is reminded that lower indices occurring after a comma designate derivatives with respect to y. Upon collecting all terms in Eq. (51), we arrive at the following equation for the correlation tensor $H^{i j}(t, \mathbf{y})$ (cf. Vainshtein 1980, 1982b; Molchanov, Ruzmaikin, and Sokoloff 1983; Kichatinov 1985; Vainshtein and Kichatinov 1986):

$$
\begin{aligned}
\partial_{t} H^{i j}-2 \eta \Delta H^{i j}= & -\left(\kappa^{k l}-\kappa^{k l}(0)\right) H_{, k l}^{i j}-\kappa_{, k l}^{i j} H^{k l}+\kappa_{, k}^{i l} H_{, l}^{k j}+\kappa_{, k}^{j l} H_{, l}^{k i} \\
& -2 \kappa_{, k}^{k l} H_{, l}^{i j}-\kappa_{, k l}^{k l} H^{i j}+\kappa_{, k l}^{i l} H^{k j}+\kappa_{, k l}^{j l} H^{k i} .
\end{aligned}
$$

Since the magnetic field is isotropic and solenoidal $\left(H_{, j}^{i j}=0\right)$, all the necessary information about its two-point correlation properties is contained in just one scalar function of $y$. The most convenient (and customary) choice is the longitudinal correlation function

$$
H_{L L}(t, y)=\frac{y^{i} y^{j}}{y^{2}} H^{i j}(t, \mathbf{y})
$$

in terms of which the correlation tensor $H^{i j}(t, \mathbf{y})$ can be expressed as follows (see Appendix A.2):

$$
H^{i j}(t, \mathbf{y})=H_{L L}(t, y) \delta^{i j}+\frac{1}{d-1} y H_{L L}^{\prime}(t, y)\left(\delta^{i j}-\frac{y^{i} y^{j}}{y^{2}}\right) .
$$

Here and below primes denote derivatives with respect to $y$. Multiplying Eq. (53) by $y^{i} y^{j} / y^{2}$ and making use of the formulas (54) and (55), and after more tedious algebra, we find that the longitudinal correlation function of the magnetic field satisfies the following evolution equation:

$$
\begin{aligned}
\partial_{t} H_{L L}= & K(y) H_{L L}^{\prime \prime}+\left(2 \frac{d-1}{y} K(y)+K^{\prime}(y)+\frac{3-d}{y} Q(y)\right) H_{L L}^{\prime} \\
+ & \frac{d-1}{y}\left(K^{\prime}(y)+Q^{\prime}(y)+\frac{d-2}{y}[K(y)-Q(y)]\right) H_{L L},
\end{aligned}
$$

where we have introduced two "renormalized diffusivities"

$$
\begin{aligned}
& K(y)=2 \eta+\kappa_{L L}(0)-\kappa_{L L}(y) \\
& Q(y)=2 \eta+\frac{\kappa_{N N}(0)-\kappa_{N N}(y)}{d-1}=2 \eta+\kappa_{L L}(0)-\frac{\kappa_{N N}(y)}{d-1} .
\end{aligned}
$$

Note that, in the incompressible case, we should have (see Appendix A.2)

$$
Q(y)=K(y)+\frac{y K^{\prime}(y)}{d-1} .
$$


Due to the realizability constraints, it is assured that $K(y)>0, Q(y)>0$ (see Appendix A.3). Eq. (56) is the configuration-space analog of the mode-coupling equation (11). While we have chosen to derive it by directly averaging the induction equation, it could also have been done by Fourier-transforming the mode-coupling equation (11). We should like to remark, however, that Eq. (56) is more convenient than the mode-coupling equation (11) for the study of general velocity correlators, such as those whose small-scale expansion is nonanalytic (as in the case of $\operatorname{Pr}<1$ ).

A further modification of Eq. (56) brings us to the quantum-mechanical analogy that was mentioned in the Introduction. Namely, we introduce the " $\psi$-function"

$$
\psi(t, y)=y^{d-1} \sqrt{K(y)} H_{L L}(t, y) \exp \left(\frac{3-d}{2} \int^{y} \mathrm{~d} y^{\prime} \frac{Q\left(y^{\prime}\right)}{y^{\prime} K\left(y^{\prime}\right)}\right)
$$

and find that it satisfies the one-dimensional Schrödinger equation,

$$
\partial_{t} \psi=\frac{1}{2 m(y)} \psi^{\prime \prime}-V(y) \psi, \quad y \geq 0
$$

which describes the quantum mechanics of a particle with variable mass ${ }^{6} m(y)=[2 K(y)]^{-1}$ in a onedimensional (or radial) potential

$$
V(y)=\frac{1}{2} K^{\prime \prime}(y)-\frac{\left[K^{\prime}(y)\right]^{2}}{4 K(y)}+\frac{3 d-5}{2 y^{2}}\left[Q(y)-y Q^{\prime}(y)\right]+\frac{(d-3)^{2}}{4 y^{2}} \frac{[Q(y)]^{2}}{K(y)} .
$$

Eq. (61) [with potential (62)] was first derived by Boldyrev and Schekochihin (1998). In the three-dimensional case and for incompressible velocity field, it reduces to the equation derived by Kazantsev (1968) (see also Kichatinov 1985; Vainshtein and Kichatinov 1986). In what follows, the general equation (61) will also be referred to as the Kazantsev equation. The expression for the potential (62) in the incompressible case becomes [see formula (59)]

$$
V(y)=\frac{d^{2}-1}{4 y^{2}} K(y)-\frac{d-2}{d-1}\left[K^{\prime \prime}(y)+\frac{d+1}{y} K^{\prime}(y)+\frac{\left[K^{\prime}(y)\right]^{2}}{(d-1) K(y)}\right] .
$$

It is this incompressible form that has mostly been considered in the literature (Ruzmaikin and Sokoloff 1981; Novikov, Ruzmaikin, and Sokoloff 1983; Artamonova and Sokoloff 1986; Kleeorin, Ruzmaikin, and Sokoloff 1986; Maslova and Ruzmaikin 1987). Note that, in 2D incompressible case, it is immediately clear from the formula (63) that the potential is repelling and hence no bound states exist.

\subsection{The Eigenvalue Problem Associated with the Kinematic Dynamo: Asymptotic Solutions}

We now consider the eigenvalue problem associated with the Kazantsev equation:

$$
-\lambda \gamma \psi=-\frac{1}{2 m(y)} \psi^{\prime \prime}+V(y) \psi, \quad y \geq 0,
$$

where, for the sake of theoretical uniformity, we measure the eigenvalues (dynamo growth rates) in terms of $\gamma$, the magnetic energy growth rate (14) that figured in Sec. 2. [In terms of the configuration-space

\footnotetext{
${ }^{6}$ The mass $m(y)$ is always positive due to the realizability constraint (A38) (see Appendix A.3).
} 
quantities, $\gamma$ is proportional to the square of the velocity shear: $\gamma=\left|\kappa_{L L}^{\prime \prime}(0)\right| / 2 A$, where $A$ is defined by the formula (22). See Appendix A.4, formula (A51).] The objective is to find the spectrum of the Schrödinger operator in the right-hand side of Eq. (64). Clearly, positive energies correspond to damped modes $(\lambda<0)$, while negative energies represent growth (the dynamo effect, $\lambda>0$ ). Therefore, the ground state of Eq. (64), if it exists, would give the desired long-time asymptotic of the magnetic-field correlation function.

At small scales, the velocity correlation tensor (50) has a Taylor expansion,

$$
\kappa^{i j}(\mathbf{y})=\kappa_{0} \delta^{i j}-\frac{1}{2} \kappa_{2}\left(y^{2} \delta^{i j}+2 a y^{i} y^{j}\right)+\cdots,
$$

and the renormalized diffusivities $K(y)$ and $Q(y)$ therefore are, to second order (see Appendix A.4),

$$
\begin{aligned}
& K(y) \simeq 2 \eta+\frac{1}{2}(1+2 a) \kappa_{2} y^{2}, \\
& Q(y) \simeq 2 \eta+\frac{1}{2} \kappa_{2} y^{2},
\end{aligned}
$$

where $a$ is the compressibility parameter. These are valid for $y \ll\left(\kappa_{0} / \kappa_{2}\right)^{1 / 2} \sim k_{\nu}^{-1}$, where $\kappa_{0}=\kappa_{L L}(0)$. Note that $(1+2 a) \kappa_{2}=\left|\kappa_{L L}^{\prime \prime}(0)\right|=2 \gamma A$ [see formula (A51)].

When $y \gg\left(\kappa_{0} / \kappa_{2}\right)^{1 / 2}$, both $K(y)$ and $Q(y)$ tend to a constant value:

$$
K(y) \simeq Q(y) \simeq 2 \eta+\kappa_{0} \simeq \kappa_{0} .
$$

Substituting these small- and large-scale asymptotics of $K(y)$ and $Q(y)$ into the expressions for the mass of our particle and for the potential it lives in [see (62)], we may gain a qualitative idea of what $m(y)$ and $V(y)$ look like. [The typical forms of the mass and potential (in 3D) are sketched in Fig. 7 and Fig. 8.] Three regions with different asymptotics are clearly pronounced: the subresistive scales, the integral scales, and the intermediate inertial range. Let us analyze the situation in each of them in turn.

The subresistive scales. These are, of course, the scales satisfying $y \ll\left(\eta / \kappa_{2}\right)^{1 / 2} \sim k_{\eta}^{-1}$. There, the mass of the particle is constant and the potential is a repelling inverse square:

$$
\begin{aligned}
& m(y) \simeq \frac{1}{4 \eta} \\
& V(y) \simeq \frac{1}{2 m(0)} \frac{d^{2}-1}{4 y^{2}} \rightarrow+\infty, \quad y \rightarrow+0 .
\end{aligned}
$$

The asymptotic solutions of the eigenvalue problem (64) in this approximation are

$$
\psi(y)=y^{(1 \pm d) / 2},
$$

where it is clearly the "+"-branch that must be picked. Indeed, it satisfies just the right boundary condition that follows from the definition of the "wave function" (60) and the requirement that $H_{L L}(t, y)$ have a positive finite value at $y=0$ (it is the total magnetic energy in the system). Note that the boundary condition could not have been satisfied had there not been a nonzero magnetic diffusivity in the problem.

The solution in this region does not depend on the value of $\lambda$, in a precise analogy to what we saw in the wave-number space [see the asymptotic (45)]. 
The integral scales. Considering the opposite end of the available range of scales, for $y \gg\left(\kappa_{0} / \kappa_{2}\right)^{1 / 2} \sim$ $k_{\nu}^{-1}$, we again find a constant (now "renormalized") mass and a repelling inverse-square potential:

$$
\begin{aligned}
& m(y) \simeq \frac{1}{2\left(2 \eta+\kappa_{0}\right)} \simeq \frac{1}{2 \kappa_{0}}, \\
& V(y) \simeq \frac{1}{2 m(\infty)} \frac{d^{2}-1}{4 y^{2}} \rightarrow+0, \quad y \rightarrow+\infty .
\end{aligned}
$$

This time, however, it is the potential that contributes subdominantly to the solution, while the eigenvalue $\lambda$ plays an important role (unless $\lambda=0$ ). The solutions that do not grow at infinity are

$$
\begin{aligned}
& \psi(y)=\exp \left(-\sqrt{\frac{\lambda \gamma}{\kappa_{0}}} y\right), \quad \lambda>0, \\
& \psi(y)=y^{-(d-1) / 2}, \quad \lambda=0, \\
& \psi(y)=\sin \left(\sqrt{\frac{|\lambda| \gamma}{\kappa_{0}}} y+\varphi\right), \quad \lambda<0 .
\end{aligned}
$$

We see from the form of the solution, as well as from the fact that the potential approaches zero from above as $y \rightarrow+\infty$, that no bound states can exist with negative $\lambda$. In fact, neither can they exist with $\lambda=0$, for it leads to a power-like decay of the wave function at best.

The inertial range. These are the scales that are equally removed both from where resistive-diffusive effects operate and from the integral scales where the details of the velocity-field correlation properties start to be felt. Namely, $\left(\eta / \kappa_{2}\right)^{1 / 2} \ll y \ll\left(\kappa_{0} / \kappa_{2}\right)^{1 / 2}$. In this regime, we obtain

$$
\begin{aligned}
& m(y) \simeq \frac{1}{(1+2 a) \kappa_{2} y^{2}}, \\
& V(y) \simeq-V_{0}, \quad \text { where } V_{0}=\frac{\kappa_{2}}{4}\left[3 d-5-\frac{(d-3)^{2}}{2(1+2 a)}\right] .
\end{aligned}
$$

We have clearly found ourselves inside the potential well, which is locally flat. An obvious estimate for the eigenvalue immediately follows: $\lambda<V_{0} / \gamma=\lambda_{\max }$, which is the same as the upper bound obtained in $\S 2.5$ [see the inequality (43)]. We find that, in $d=3$, the potential well always extends below zero (so $V_{0}>0$ ), while in $d=2, V_{0} \leq 0$ for $a \leq-1 / 4$.

Eq. (64) in this regime takes the form

$$
\psi^{\prime \prime}+\frac{\lambda_{\max }-\lambda}{A y^{2}} \psi=0,
$$

where $A$ is defined by the formula (22) and it can be easily verified that $(1+2 a) \kappa_{2}=2 \gamma A$. The effective potential in Eq. (79) is of the inverse-square kind, as advertised in the Introduction ( $§ 1.3)$. This equation is, of course, the configuration-space version of Eq. (40) in the inertial-scale limit. Its solutions are, expectedly,

$$
\psi(y)=c_{1} y^{1 / 2+\sqrt{\left(\lambda-\lambda_{0}\right) / A}}+c_{2} y^{1 / 2-\sqrt{\left(\lambda-\lambda_{0}\right) / A}}
$$

where $\lambda_{0}=\lambda_{\max }-A / 4$ is defined as it was in $\S 2.5$ [see formula (28)]. 


\subsection{Asymptotic Matching: The Dynamo Growth Rates in the Long-Time Limit}

In order to complete the asymptotic solution of the problem, one must now proceed to properly match the solutions we have obtained in the three asymptotic scale ranges described above.

Matching the inertial range solution with the subresistive one does not present any difficulty in principle. Indeed, we saw in Sec. 2 that, in the $\mathbf{k}$ space, an analytic solution could be obtained that is valid both in the resistive and inertial ranges. The issue of matching the inertial-range and the large-scale solutions is more troublesome. The appropriate matching procedure depends on the full functional form of the potential at the velocity-field scales and must typically be performed assuming a particular form of the velocity correlator (cf. Artamonova and Sokoloff 1986; Kleeorin, Ruzmaikin, and Sokoloff 1986).

In this section, we propose a simple way to match the asymptotic solutions obtained in $\S 3.2$ and calculate the dynamo growth rates in the long-time limit. Our method is based on the assumption that the particular way the Kazantsev particle mass and potential are regularized at small and large scales does not play an essential part in determining the dynamo growth rates. This assumption holds for such forms of the velocity correlation function that ensure that the potential well be located at small (inertial) scales and that the potential (62) have no relevant structure at the integral scales. In other words, our model quantum-mechanical particle must be "localized" at small scales in order for the structure of the velocity correlations to be unimportant. If this is satisfied, the dynamo growth rates in the long-time limit can be calculated in a universal way. Let us explain how this can be accomplished.

Although, as we have noted, matching the subresistive and the inertial-range solutions is no problem in principle, it does require a considerable amount of algebra (Kleeorin, Ruzmaikin, and Sokoloff 1986). The formal difficulty lies in the fact that $\psi(y)$ and $\psi^{\prime}(y)$ at $y \sim\left(\eta / \kappa_{2}\right)^{1 / 2}$ are of different orders in $\eta$. The matching must therefore be carried out in two successive orders of the asymptotic expansion. This difficulty can be circumvented in the following way, suggested by Landau's discussion of the quantum mechanics in the inverse-square potential (Landau and Lifschitz 1977). Assuming that the particular form of the smallscale regularization is not essential for choosing the right solution in the inertial range, let us pick a small $y_{\eta} \sim\left(\eta / \kappa_{2}\right)^{1 / 2}$, extend the inertial-range forms of the mass and potential [formulas (77) and (78)] down to this point (to all $y>y_{\eta}$ ), and replace the actual values of $m(y)$ and $V(y)$ at $y<y_{\eta}$ by constants equal to their values at $y=y_{\eta}$ (thus taking "a shortcut" to the "wall" at $y=0$ ). Let us then solve the resulting eigenvalue problem in both regions and match the solutions at $y=y_{\eta}$. We will then carry out a similar procedure to match the inertial-range and the large-scale solutions at some $y_{0} \sim\left(\kappa_{0} / \kappa_{2}\right)^{1 / 2}$ (see below). Eventually, we must take the limit $y_{\eta} \rightarrow 0$ and $y_{0} \rightarrow \infty$ and determine the eigenvalues $\lambda$ from the requirement that the matching relations at $y_{\eta}$ and $y_{0}$ be consistent with each other in this limit.

Let us empahsize that our matching procedure corresponds to a particular reasonable choice of the small- and large-scale regularization of the Kazantsev quantum mechanics. Its universal applicapility hinges on the assumption that the specific form of such regularization is inessential.

The proper solution in the interval $0<y<y_{\eta}$ is $\psi(y)=\sin \left(\gamma_{\eta} y / y_{\eta}\right)$, where $\gamma_{\eta}=\sqrt{\left(\lambda_{\max }-\lambda\right) / A}$ (we assume, of course, that $\left.\lambda<\lambda_{\max }\right)$. The solution at $y>y_{\eta}$ is given by the formula (80). Matching $\psi\left(y_{\eta}\right)$ and $\psi^{\prime}\left(y_{\eta}\right)$, we get, for $\lambda>\lambda_{0}$,

$$
\frac{c_{2}}{c_{1}}=\frac{s_{1}-\gamma_{\eta} \cot \gamma_{\eta}}{\gamma_{\eta} \cot \gamma_{\eta}-s_{2}} y_{\eta}^{s_{1}-s_{2}}, \quad \lambda_{0}<\lambda<\lambda_{\max }
$$

Here we have denoted $s_{1,2}=1 / 2 \pm \sqrt{\left(\lambda-\lambda_{0}\right) / A}$. For $\lambda<\lambda_{0}$, it is convenient to write the inertial-range 
solution (80) as

$$
\psi(y)=c y^{1 / 2} \sin \left[\sqrt{\frac{\delta \lambda}{A}} \ln \left(\frac{y}{y_{\eta}}\right)+\chi\right], \quad \delta \lambda=\lambda_{0}-\lambda>0 .
$$

The matching determines the value of the phase:

$$
\cot \chi=\frac{\gamma_{\eta} \cot \gamma_{\eta}-1 / 2}{\sqrt{\delta \lambda / A}}, \quad \text { where } \quad \gamma_{\eta}=\frac{1}{2} \sqrt{1+\frac{4 \delta \lambda}{A}} .
$$

Let us now employ the same device to match the inertial-range and the large-scale solutions. Namely, we now pick some $y_{0} \sim\left(\kappa_{0} / \kappa_{2}\right)^{1 / 2}$, formally extend the validity of the inertial-range asymptotics of $m(y)$ and $V(y)$ to all $y<y_{0}$, while at $y>y_{0}$, let $m(y)=1 / 2 \kappa_{0}$ and $V(y)=0$. The solution in the latter region is the large-scale solution (74) or (76) (depending on the sign of $\lambda$ ). For convenience, let us take $y_{0}=$ $\left(\kappa_{0} / \gamma A\right)^{1 / 2}$, whence, in the formulas (74) and (76), we have, by definition, $\lambda \gamma / \kappa_{0}=\lambda / A y_{0}^{2}=\left(\gamma_{0} / y_{0}\right)^{2}$, where $\gamma_{0}=\sqrt{|\lambda| / A}$. In order to match $\psi\left(y_{0}\right)$ and $\psi^{\prime}\left(y_{0}\right)$, we take the asymptotic solutions appropriate for various values of $\lambda$ : for $\lambda>\lambda_{0}$, the formulas (80), (74), and (76) imply

$$
\begin{aligned}
& \frac{c_{1}}{c_{2}}=-\frac{s_{2}+\gamma_{0}}{s_{1}+\gamma_{0}} y_{0}^{s_{2}-s_{1}}, \quad \lambda>\lambda_{0} \& \lambda \geq 0, \\
& \frac{c_{1}}{c_{2}}=\frac{s_{2}-\gamma_{0} \cot \left(\gamma_{0}+\varphi\right)}{\gamma_{0} \cot \left(\gamma_{0}+\varphi\right)-s_{1}} y_{0}^{s_{2}-s_{1}}, \quad \lambda_{0}<\lambda<0 ;
\end{aligned}
$$

for $\lambda<\lambda_{0}$, we use the formula (82) instead of (80) and get

$$
\begin{aligned}
\cot \left[\sqrt{\frac{\delta \lambda}{A}} \ln \left(\frac{y_{0}}{y_{\eta}}\right)+\chi\right] & =-\frac{\gamma_{0}+1 / 2}{\sqrt{\delta \lambda / A}}, \quad 0 \leq \lambda<\lambda_{0}, \\
\cot \left[\sqrt{\frac{\delta \lambda}{A}} \ln \left(\frac{y_{0}}{y_{\eta}}\right)+\chi\right] & =\frac{\gamma_{0} \cot \left(\gamma_{0}+\varphi\right)-1 / 2}{\sqrt{\delta \lambda / A}}, \quad \lambda<\lambda_{0} \& \lambda<0,
\end{aligned}
$$

where $\delta \lambda=\lambda_{0}-\lambda$. Note that the relations (84) and (86) smoothly extend to the case of $\lambda=0$ (where $\left.\gamma_{0}=0\right)$, which does not have to be treated separately.

We are now in a position to find the dynamo growth rates. In the matching conditions derived above, we take the limit $y_{\eta} / y_{0} \rightarrow 0$ and determine the allowed values of $\lambda$ from the consistency requirements.

The case $\lambda>\lambda_{0} \& \lambda \geq 0$ (growth). For these values of $\lambda$, the relations (81) and (84) that ensure proper matching of the inertial-range solution at dissipative and integral scales respectively are incompatible in the limit $y_{\eta} / y_{0} \rightarrow 0$ unless

$$
\left(\gamma_{0}+s_{1}\right)\left(\gamma_{\eta} \cot \gamma_{\eta}-s_{2}\right)=0
$$

This is a transcendental equation on $\lambda$, which is readily seen to have no solutions. Thus, no positive growth rates that are larger than $\lambda_{0} \gamma$ are allowed.

Note that, in $2 \mathrm{D}$, while we have $\lambda_{0} \leq 0$, a rudimentary potential well exists for some values of the compressibility parameter: in $\S 3.2$, we already pointed out that $\lambda_{\max }>0$ for $a>-1 / 4$. We see now that this well has turned out to be too shallow to sustain a bound state with $\lambda \geq 0 \geq \lambda_{0}$. 
The case $0 \leq \lambda<\lambda_{0}$ (growth). In this case, we have to make sure that the relations (83) and (86) are consistent with each other in the limit $y_{0} / y_{\eta} \sim \operatorname{Pr}^{1 / 2} \rightarrow \infty$. If $\delta \lambda=\lambda-\lambda_{0}$ remains finite under this limit, the left-hand side of Eq. (86) has no definite limit, while its right-hand side is equal to a finite constant. The matching is therefore impossible. On the other hand, if we assume that $\delta \lambda \rightarrow+0$ as $y_{0} / y_{\eta} \rightarrow \infty$, the right-hand sides of both relations (86) and (83) tend to infinity, whence $\chi=\pi m, m \in \mathbb{Z}$, and

$$
\delta \lambda=\frac{A \pi^{2} n^{2}}{\left[\ln \left(y_{0} / y_{\eta}\right)\right]^{2}} \simeq \frac{A \pi^{2} n^{2}}{\left[\ln \left(\operatorname{Pr}^{1 / 2}\right)\right]^{2}} \ll 1, \quad n=1,2, \ldots
$$

Clearly, it does not matter here what the particular definitions of $y_{0}$ and $y_{\eta}$ are: multiplying any given such definitions by finite constants leads to a finite, and hence subdominant, correction in the denominator of the expression (89).

The expression (89) reproduces the result that was obtained in $\S 2.5$ [see formula (49)] by requiring the magnetic-energy spectrum to vanish at some particular ("smallish") wave number. Within our model, we have now demonstrated that this result gives a valid long-time asymptotic for the kinematic dynamo problem. The key to understanding this coincidence lies in the fact that the magnetic-field correlation function decays exponentially at large scales [asymptotic solution (74)]. Due to the solenoidality of the magnetic field, such exponential tail implies that $H(k)$ must vanish at $k=0$ [see Appendix A.2, formula (A27)]. Imposing this as a boundary condition on the small-scale spectrum (44) necessarily implies (49) or (89).

The solution we have thus obtained is relevant in three dimensions, where $\lambda_{0}>0$. Note that formula (89) can be used to estimate the critical value of the Prandtl number that has to be exceeded in order for the dynamo effect to occur (i.e., for the growth rate to be positive, $\lambda=\lambda_{0}-\delta \lambda>0$ ): $\operatorname{Pr}_{c} \sim \exp \left(2 \pi \sqrt{A / \lambda_{0}}\right)$. For the incompressible case, $\operatorname{Pr}_{c} \sim 26$; for the irrotational case, $\operatorname{Pr}_{c} \sim 17000$. It must be clear, of course, that such critical values are not to be taken as anything more than rough estimates, for they have been deduced from the formula (89), which is strictly valid only when $\delta \lambda \ll 1$. Numerical studies of the excitation threshold for the Kazantsev equation give (in the incompressible case) values of the critical Prandtl number (or critical magnetic Reynolds number, which, for a one-scale velocity field, is the same thing) about $\operatorname{Pr}_{c} \sim 53$ (Novikov, Ruzmaikin, and Sokoloff 1983; Maslova and Ruzmaikin 1987).

The case $\lambda_{0}<\lambda<0$ (damping). Here we must reconcile the relations (85) and (81) in the limit $y_{\eta} / y_{0} \rightarrow$ 0 . In view of the incompatibility of Eq. (88), this can only be done if

$$
\gamma_{0} \cot \left(\gamma_{0}+\varphi\right)-s_{1}=0
$$

This fixes the value of the phase $\varphi$ for any applicable $\lambda$. No further constraint ensues for $\lambda$ lying in the interval $\lambda_{0}<\lambda<0$. Provided this interval is nonempty (as is the case in two dimensions, unless $a=0$ ), a continuous spectrum of eigenvalues exists and fills the entire interval. Since the upper bound of the spectrum is zero, a power-like decay of the magnetic fluctuations should be expected.

The case $\lambda<\lambda_{0} \& \lambda<0$ (damping). In this case, just like in the case of $0 \leq \lambda<\lambda_{0}$, assuming that $\delta \lambda$ remains finite (or becomes large) as $y_{0} / y_{\eta} \rightarrow \infty$ leads to incompatibility between the right- and left-hand sides of Eq. (87): on the right-hand side, there must be a finite constant, while the left-hand side has no definite limit. If $\lambda_{0}>0(3 \mathrm{D})$, this is the only possibility. Thus, if $\lambda_{0}$ is positive, negative values of $\lambda$ are ruled out.

If $\lambda_{0} \leq 0$ (2D), it is possible to have $\delta \lambda \rightarrow+0$ as $y_{0} / y_{\eta} \rightarrow \infty$. Again, the argument that led to the solution (89) remains valid. However, this solution is not relevant because we have already discovered a 
continuous spectrum between $\lambda_{0}$ and 0 . It is the resulting power-like decay that will determine the long-time behavior of the magnetic fluctuations. The case of $\lambda_{0}=0$, which occurs in 2D for $a=0$ (the "quasiequilibrium" case we noted in $\S 2.3)$, appears to be exceptional in this context, for then the interval $\left(\lambda_{0}, 0\right)$ is empty and the only applicable solution is, in fact, (89) (where now $\lambda<\lambda_{0}=0$ ). Namely, we have $\lambda \simeq-A \pi^{2} /\left[\ln \left(\operatorname{Pr}^{1 / 2}\right)\right]^{2} \rightarrow-0$, which should also result in a power-like temporal decay law in the limit of vanishing resistivity $(\eta \rightarrow+0)$.

Remark on the case $\lambda=\lambda_{0}$. When $\lambda=\lambda_{0}$, we have $s_{1}=s_{2}=1 / 2$ and the solution (80) appears to be degenerate. This degeneracy is spurious. In fact, the power-like solutions (80) are asymptotics of the modified Bessel functions $I_{\sqrt{\left(\lambda-\lambda_{0}\right) / A}}$ and $K_{\sqrt{\left(\lambda-\lambda_{0}\right) / A}}$. This can be seen if the Kazantsev eigenvalue problem (64) is solved in the inertial range with next-order correction terms in the Taylor expansions of $K(y)$ and $Q(y)$ included [see Eq. (C8) in Appendix C below]. When $\lambda=\lambda_{0}$, we should therefore use, instead of the asymptotic solution (80),

$$
\psi(y)=y^{1 / 2}\left(c_{1}+c_{2} \ln y\right) .
$$

The matching at $y_{\eta}$ and $y_{0}$ can be carried out in quite the same fashion as before. The consistency conditions as $\ln \left(y_{0} / y_{\eta}\right) \rightarrow \infty$ are given by Eq. (88) if $\lambda_{0} \geq 0$ (still incompatible) and Eq. (90) if $\lambda_{0}<0$ (can always be satisfied).

\subsection{Discussion}

Thus, assuming that the particular form of the large-scale regularization of the Kazantsev quantum mechanics is unimportant, or, in other words, that the Kazantsev quantum-mechanical particle is "localized" at small (inertial) scales, we have been able to make progress in our investigation of the long-time asymptotic behavior of the passive magnetic fluctuations. In $2 \mathrm{D}$, a continuous spectrum of negative $\lambda$ 's exists, which indicates a power-like temporal decay. Its exact nature can be discovered only by solving the time-dependent dynamo equation (56). In $3 \mathrm{D}$, a universal dynamo growth rate has been found, which asymptotically approaches $\lambda_{0} \gamma$ from below, as $\operatorname{Pr} \rightarrow \infty$ (the convergence is square-logarithmic).

The exact quantitative criterion that would clearly define the class of velocity correlators within which such universality with respect to the large-scale regularization exists, is unknown. Due to the complicated structure of the potential (62), it appears to be very hard to determine. On the other hand, no examples of realizable ${ }^{7}$ velocity correlators for which the dynamo growth rate is essentially determined by the largescale properties of the velocity field are available. As we already mentioned in the Introduction, numerical solution of the Kazantsev eigenvalue problem (64) for various plausible forms of the velocity correlator (see Novikov, Ruzmaikin, and Sokoloff 1983; Artamonova and Sokoloff 1986; Maslova and Ruzmaikin 1987, and Appendix C), including the one corresponding to the Kolmogorov turbulent spectrum (Kulsrud and Anderson 1992), did not reveal any nonuniversal behavior of the second-order statistics of the magnetic field. Thus, the validity of the universal results presented in this section appears quite robust.

A tentative explanation for such robustness could be given along the following lines. The mass $m(y)$ of the Kazantsev particle at integral scales tends to the constant finite value $m(\infty) \simeq 1 / 2 \kappa_{0}$. At small (inertial) scales, however, it sharply increases and, at the resistive scales, reaches the asymptotically infinite

\footnotetext{
${ }^{7}$ I.e., those whose Fourier transforms are correlation functions (see Appendix A.1).
} 
value $m(0)=1 / 4 \eta$ (for $\eta \rightarrow+0$ ). Thus, the particle is impelled to slide toward the small scales not just by the potential, but also by its own variable mass: it is "heavier at small scales." This leads to the large-scale effects being marginalized even if the value of the potential in the inertial range, $V(y) \sim-V_{0}$, is not, in fact, its global (or even local) minimum. Note that, while the latter situation has not been encountered in the previous studies of the incompressible case, it turns out to be quite common when the advecting flow possesses some degree of compressibility (see Appendix C).

It remains to be seen if examples of velocity correlators can be constructed for which the second-order statistics depart from the universal solutions obtained above. The issue of whether higher-order statistics may be more vulnerable in this respect is also open to investigation. At the current level of understanding of the two-point kinematic dynamo, we may conclude that, while the nonuniversality of the long-time solutions due to large-scale effects remains no less of a possibility than it was when first hinted at by Kazantsev (1968), the results obtained assuming small-scale universality appear quite reliable for practical purposes.

\section{Where Do We Go From Here?}

The main physical conclusion of the kinematic theory developed above is that, at the moment when the nonlinear effects become important, the magnetic energy is concentrated at the smallest scales that it is able to reach at the exponential rate $\sim \gamma$ during the kinematic stage of the dynamo. An ascending power spectrum $\sim k^{3 / 2}$ extends through the magnetic inertial range up to the wavenumber where the peak of the spectrum is located at the time the nonlinearity sets in.

It is important to understand that the second-order (spectral) theory developed here does not contain all of the statistical information necessary to determine the condition for the onset of the nonlinearity. Indeed, the nonlinear back-reaction effect is controlled by the Lorentz tension force $\propto(\mathbf{B} \cdot \nabla) \mathbf{B}$ in the momentum equation of the fluid (in the incompressible case). The second-order statistics of this quantity are fourth order in the magnetic field. Importantly, the statistics of $(\mathbf{B} \cdot \nabla) \mathbf{B}$ are determined not just by the magnitude of the magnetic field and by its overall characteristic scale, but also by the structure of the field, namely, by the characteristic scale of its variation along itself. This issue was thoroughly investigated by Ott and coworkers (see review by Ott 1998), Cattaneo (1997, 1999), and by Schekochihin et al. (2002a) (see also references therein). The result is that the parallel scale does not decay with the overall characteristic scale, but stays at values comparable to the velocity scale $k_{\nu}^{-1}$. The small-scale field is arranged in a pattern of randomly oriented long (of characteristic size $\sim k_{\nu}^{-1}$ ) folds, whithin which the field undergoes rapid transverse spatial oscillations of its direction. The implication is that the condition for the onset of the nonlinearity is the approximate equalization of the magnetic-field energy and the energy of the smallest turbulent eddies.

Once this has occurred, a nonlinear theory is needed to describe futher evolution of the field. No such theory exists as yet. However, there does exist a fair amount of numerical evidence, which, along with the heuristic physical insight, can be used as guidance in mapping out the tentative strategies of picking up where the kinematic theory left off and building on its results. Resolving three main issues appears to be the first order of business at this point.

Nonlinear saturation at subviscous scales. The onset of the nonlinear regime can occur under two distinct sets of circumstances, depending on the magnitude of the initial seed field and on the magnetic Prandtl number. As we saw in the preceding sections, the growth of the field strength and the refinement of its scale proceed in parallel. With the seed field assumed to be concentrated at velocity scales $\sim k_{\nu}^{-1}$, the 
time it takes for the dynamo to switch from the diffusion-free to the resistive regime is of order $\sim \gamma^{-1} \log \operatorname{Pr}$. Given a very weak seed field, the dynamo will still be kinematic at the time of this transition. The input state for the nonlinear theory will then be that with the small-scale magnetic-energy spectrum extending through the entire subviscous scale range and peaked at the resistive scale $\sim k_{\eta}^{-1}$ (see $\S 2.4, \S 2.5$ and Sec. 3). Alternatively, it is conceivable that the initial field should be sufficiently strong for the nonlinear regime to commence before the resistive scale is reached (Kulsrud 2000) (see $\S 2.3$ ). One wonders then whether the nonlinear back reaction is capable of arresting all further refinement of the field scale. Although the answer to this question is in the negative, it appears that the spreading of the magnetic excitation into the remainder of the subviscous range available to it proceeds at a much slower pace than in the kinematic regime: most likely, at the resistive time scale (Kinney et al. 2000a,b; Schekochihin et al. 2002b). It may be possible to study the evolution of the magnetic spectrum through this initial nonlinear stage in a theoretical framework that is substantially simpler than the full nonlinear MHD. Namely, the action of the fluid motions on the small-scale magnetic fields can be modelled by balancing the viscous and magnetic stresses, rather than by solving the full Navier-Stokes equation with Lorentz back reaction (the so-called Stokes model). An "intermediate" small-scale nonlinear theory emerges from such an approach that is a direct extension of the kinematic theory elaborated above (Kinney et al. 2000a; Schekochihin et al. 2002b); an exactly solvable model of such a regime (without diffusion) was constructed by Boldyrev (2001).

Plasma damping mechanisms. A parallel question is how well the Spitzer resistive-diffusive smallscale regularization that is a part of the standard MHD paradigm describes what really happens at subviscous scales in the high-Prandtl-number astrophysical plasmas. It must be realized that, while the resistive scales $k_{\eta}^{-1}$ that obtain by substituting the ISM or protogalactic-plasma parameters into the Spitzer formula for the magnetic diffusivity $\eta$ may be 7 to 11 decades below the viscous scale $k_{\nu}^{-1}$, the mean free path of the particles that make up these plasmas is substantially larger (the simplest estimate gives $\ell_{\mathrm{mfp}} \sim \operatorname{Re}^{-1 / 4} k_{\nu}^{-1}$, i.e., only about 10 times shorter than the viscous scale). The MHD approximation is not strictly valid at scales below the mean free path, and one must allow that a number of plasma damping mechanisms may supercede the Ohmic resistive dissipation (Vainshtein 1976; Kulsrud and Anderson 1992; Kulsrud et al. 1997b). These include, most importantly, the ambipolar damping that is present due to the partial ionization of the ISM (Kulsrud and Anderson 1992; Chandran 1998; Subramanian 1997, 1998, 1999; Brandenburg and Subramanian 2000) as well as the kinetic effects due to the Braginskii (1965a) tensor viscosity and magnetic diffusivity (Malyshkin 2001) and to Landau damping of magnetic fluctuations (Kulsrud et al. 1997b). We note that, while all of these effects are nonlinear in the magnetic field, the kinetic effects depend solely on the direction, not the magnitude, of the field, which only needs to be sufficient to magnetize the ions - a condition that is satisfied in the astrophysical plasmas in question already for very weak fields, which are otherwise passive. Treatment of these effects again appears possible by means of certain amendments to the kinematic theory developed here (cf. Kulsrud and Anderson 1992; Subramanian 1997, 1998, 1999; Schekochihin and Kulsrud 1998; Malyshkin 2001). Note that, in view of the possibility of other-than-resistive small-scale regularization, the study of the onset of back reaction in the diffusion-free dynamo, as discussed in the previous paragraph, acquires additional importance.

Growth of large-scale magnetic fields. Finally, the most important - and difficult to resolve - issue is that of the feasibility of some form of nonlinear transfer of the magnetic energy accumulated at small scales toward large scales: the inverse cascade. Two distinct directions of inquiry must be clearly identified here. 
First, as we mentioned in $\S 1.1$, it has long been known that the presence of a helical component in the fluid turbulence leads to exponential growth of the large-scale magnetic field in the kinematic regime (the $\alpha$ effect). In the high-Prandtl-number astrophysical plasmas, this growth is much slower than that of the small-scale fluctuations. Once the latter have entered the nonlinear regime, they are expected to substantially modify the $\alpha$ effect, almost certainly suppressing it to some extent. The magnitude of this suppression is a subject of an ongoing debate, various estimates ranging from extreme quenching with suppression factors as large as $\mathrm{R}_{m}$ to moderate order-one reductions (see, e.g, discussion in a recent series of papers by Field, Blackman, and Chou 1999; Blackman and Field 1999, 2000; Chou 2001a; Field and Blackman 2001, and references therein). While none of the existing theories of the nonlinear $\alpha$ effect is universally agreed on, it appears safe to expect that (i) helicity does lead to some growth of the large-scale magnetic field; (ii) this growth is slowed down in a nonlinear fashion by the accumulating small-scale magnetic fluctuations. At large Prandtl numbers, the suppression effect may be very large, making impossible any growth of the large-scale fields at time scales faster than the resistive. The kinematic $\alpha$-effect growth rate, which is already about 10000 times smaller than that of the small-scale dynamo, is thus further reduced by a factor possibly as large as $\mathrm{R}_{m}$. In the context of the ISM's nearly perfect conductivity, such a dynamo would be quite prohibitively slow and could not explain the observed galactic magnetic field. [For some numerical evidence regarding these processes, we address the reader to papers by Meneguzzi, Frisch, and Pouquet (1981); Cattaneo and Hughes (1996); Brandenburg (2001); Maron and Blackman (2001). However, none of these simulations has enough resolution to be conclusive with regard to the high-Prandtl-number plasmas.]

The other avenue of investigation, which we announced in $\S 1.1$, is to look for the possibility of nonhelical inverse cascade leading to eventual equipartition of the magnetic and kinetic energies at all scales including the large. Indeed, such an eventual equipartition appears eminently plausible at the first glance, since it corresponds to the steady-state spectra that follow from Kolmogorov-style theories based on the view of the MHD turbulence as resulting from nonlinear interactions of Alfvén waves (Iroshnikov 1964; Kraichnan 1965; Goldreich and Sridhar 1995, 1997, - in this context, the outcome of the ongoing controversy over the exact form of the saturated equipartition spectrum is immaterial). However, several recent numerical simulations with $\operatorname{Pr}>1$ and no externally imposed dc background field have cast serious doubt on the possibility of the saturated equipartition state (Kinney et al. 2000a,b; Chou 2001b; Maron, Cowley, and McWilliams 2002; Schekochihin et al. 2002b). The questions of what the saturated magnetic spectrum is and whether the magnetic energy at the large scales can be shown to achieve values sufficient to give rise to an adequate theory of the observed galactic field, remain unresolved.

We are grateful to J. A. Krommes who read an earlier manuscript of this work. Both the substance and the style of the presentation have benefited from his suggestions. We would also like to thank E. Blackman, S. Cowley, J. Maron, V. Pariev, V. Rytchkov, D. Uzdensky, and the anonymous referee for useful comments. This work was supported in part by the U. S. Department of Energy under Contract No. DE-AC02-76-CHO3073 .

\section{A. CORRELATION FUNCTIONS OF ISOTROPIC RANDOM FIELDS AND OTHER MATTERS}

In this Appendix, we provide a set of useful facts and formulas that are frequently needed in turbulence calculations such as those of Section 2 and Section 3. We will work with a random field $u^{i}$, which we will often refer to as the velocity field. However, all the results of this Appendix also hold for the magnetic 
field $B^{i}$ provided solenoidality constraint is properly implemented. We find it most convenient to work in $d$ dimensions. For $d=3$, many of the results below can be found in the lucid and comprehensive chapter on the mathematical description of turbulence by Monin and Yaglom (1975).

This Appendix is organized as follows. In $\S$ A.1, we provide the transformation formulas that relate the correlation functions of isotropic fields in configuration and wave-number (Fourier) spaces. These impose certain constraints on the classes of functions in the $\mathbf{x}$ space that are proper correlation functions. In $\S$ A.2, the additional constraints arising from solenoidal and potential nature of the fields are explained. In $\S$ A.3, the so-called realizability conditions are discussed that determine some essential features of the theories dealing with isotropic random fields. In $\S$ A.4, we give some relations between the small-scale-expansion coefficients of the two-point correlation functions in the $\mathbf{x}$ space and their counterparts in the $\mathbf{k}$ space. These relations provide a bridge between the formalisms of Sec. 2 and Sec. 3 and are also needed to put the results of this paper and the one-point statistical results published earlier (Boldyrev and Schekochihin 2000; Schekochihin and Kulsrud 2001; Boldyrev 2000, 2001; Schekochihin et al. 2002a) in a unified theoretical framework. Finally, in $\S$ A.5, we list the angle-integration identities that were used in the derivations of Sec. 2 .

\section{A.1. Isotropic Fourier Transforms}

We will employ the usual Fourier-transform conventions:

$$
\begin{aligned}
& u^{i}(\mathbf{k})=\int \mathrm{d}^{d} x e^{-i \mathbf{k} \cdot \mathbf{x}} u^{i}(\mathbf{x}) \quad \text { (direct), } \\
& u^{i}(\mathbf{x})=\int \frac{\mathrm{d}^{d} k}{(2 \pi)^{d}} e^{i \mathbf{k} \cdot \mathbf{x}} u^{i}(\mathbf{k}) \quad \text { (inverse). }
\end{aligned}
$$

In this Appendix, we are not concerned with time dependence of our fields and concentrate purely on their properties arising from the assumptions of spatial homogeneity, isotropy, and mirror invariance. Under this set of assumptions, we may define the correlation functions of the field $u^{i}$ as follows: in the configuration space,

$$
\begin{aligned}
\left\langle u^{i}(\mathbf{x}) u^{j}\left(\mathbf{x}^{\prime}\right)\right\rangle & =\kappa^{i j}\left(\mathbf{x}-\mathbf{x}^{\prime}\right), \\
\kappa^{i j}(\mathbf{y}) & =\kappa_{L L}(y) \delta^{i j}-\left[\kappa_{L L}(y)-\frac{\kappa_{N N}(y)}{d-1}\right]\left(\delta^{i j}-\frac{y^{i} y^{j}}{y^{2}}\right),
\end{aligned}
$$

where $\kappa_{L L}(y)$ and $\kappa_{N N}(y)$ are called the longitudinal and the transverse correlation functions for obvious reasons. In the Fourier space,

$$
\begin{aligned}
\left\langle u^{i}(\mathbf{k}) u^{j}\left(\mathbf{k}^{\prime}\right)\right\rangle & =(2 \pi)^{d} \delta\left(\mathbf{k}+\mathbf{k}^{\prime}\right) \kappa^{i j}(\mathbf{k}), \\
\kappa^{i j}(\mathbf{k}) & =\kappa(k) \delta^{i j}+\tilde{\kappa}(k) \frac{k_{i} k_{j}}{k^{2}} .
\end{aligned}
$$

The correlation tensors $\kappa^{i j}(\mathbf{y})$ and $\kappa^{i j}(\mathbf{k})$ defined above are Fourier transforms of one another:

$$
\kappa^{i j}(\mathbf{y})=\int \frac{\mathrm{d}^{d} k}{(2 \pi)^{d}} e^{i \mathbf{k} \cdot \mathbf{y}} \kappa^{i j}(\mathbf{k}) .
$$

Let us substitute (A6) into the above formula and find that

$$
\kappa^{i j}(\mathbf{y})=\delta^{i j} \int \frac{\mathrm{d}^{d} k}{(2 \pi)^{d}} e^{i \mathbf{k} \cdot \mathbf{y}} \kappa(k)-\frac{\partial^{2}}{\partial y^{i} \partial y^{j}} \int \frac{\mathrm{d}^{d} k}{(2 \pi)^{d}} e^{i \mathbf{k} \cdot \mathbf{y}} \frac{\tilde{\kappa}(k)}{k^{2}} .
$$


The $d$-dimensional Fourier transforms of the isotropic (radial) functions can be found with the aid of the following handy formula (see, e.g., Stein 1993):

$$
\int \mathrm{d} \Omega_{d} e^{i \mathbf{k} \cdot \mathbf{y}}=(2 \pi)^{d / 2} \frac{J_{(d-2) / 2}(k y)}{(k y)^{(d-2) / 2}}=S_{d} \Phi_{d}(k y),
$$

where $\mathrm{d} \Omega_{d}$ is the $d$-dimensional angular differential, and $S_{d}=\int \mathrm{d} \Omega_{d}=2 \pi^{d / 2} / \Gamma(d / 2)$ is the area of the unit sphere in $d$ dimensions. We have defined the function

$$
\Phi_{d}(z)=\Gamma(d / 2) \frac{J_{(d-2) / 2}(z)}{(z / 2)^{(d-2) / 2}}
$$

which is the kernel of the "isotropic Fourier transform" and has many agreeable properties. Two of them will be particularly important for us: $\Phi_{d}(z)$ is analytic, its Taylor expansion for small $z$ being

$$
\Phi_{d}(z \rightarrow 0)=1-\frac{z^{2}}{2 d}+\frac{z^{4}}{8 d(d+2)}+\cdots ;
$$

and $\Phi_{d}(z)$ satisfies the following differential recursion relation:

$$
\left(\frac{1}{z} \frac{\partial}{\partial z}\right)^{n} \Phi_{d}(z)=(-1)^{n} 2^{-n}\left[\left(\frac{d}{2}\right)_{n}\right]^{-1} \Phi_{d+2 n}(z),
$$

where $(d / 2)_{n}=\Gamma(n+d / 2) / \Gamma(d / 2)$ is the Pochhammer symbol. Note also that $\Phi_{2}(z)=J_{0}(z)$ and $\Phi_{3}(z)=$ $(\sin z) / z$.

The isotropic Fourier transform is then

$$
\int \frac{\mathrm{d}^{d} k}{(2 \pi)^{d}} e^{i \mathbf{k} \cdot \mathbf{y}} \kappa(k)=\frac{S_{d}}{(2 \pi)^{d}} \int_{0}^{\infty} \mathrm{d} k k^{d-1} \Phi_{d}(k y) \kappa(k) .
$$

A similarly defined transform sometimes appears in the literature on special functions under the name of Bochner transform. After some straightforward manipulations, we obtain [from (A4) and (A8) by way of (A12)]

$$
\begin{gathered}
\kappa_{L L}(y)=\frac{S_{d}}{(2 \pi)^{d}} \int_{0}^{\infty} \mathrm{d} k k^{d-1}\left[\Phi_{d}(k y)[\kappa(k)+\tilde{\kappa}(k)]-\frac{d-1}{d} \Phi_{d+2}(k y) \tilde{\kappa}(k)\right], \\
\kappa_{N N}(y)=(d-1) \frac{S_{d}}{(2 \pi)^{d}} \int_{0}^{\infty} \mathrm{d} k k^{d-1}\left[\Phi_{d}(k y) \kappa(k)+\frac{1}{d} \Phi_{d+2}(k y) \tilde{\kappa}(k)\right] .
\end{gathered}
$$

We may also define the energy (spectrum) function

$$
\begin{aligned}
& I(k)=\kappa^{i i}(\mathbf{k})=d \kappa(k)+\tilde{\kappa}(k), \\
& I(y)=\kappa^{i i}(\mathbf{y})=\kappa_{L L}(y)+\kappa_{N N}(y)=\frac{S_{d}}{(2 \pi)^{d}} \int_{0}^{\infty} \mathrm{d} k k^{d-1} \Phi_{d}(k y) I(k) .
\end{aligned}
$$

The importance of such formulas as (A14), (A15), and (A17), lies in that they impose constraints on what functions in the configuration space may in fact be proper correlation functions. Thus, for example, since the k-space spectrum $I(k)$ must clearly be positive for all $k$ and be properly cut off at small scales, formula (A17) stipulates that only an isotropic Fourier transform of such a function can be an $\mathrm{x}$-space correlation function of $u^{i}$.

Note also that the analyticity of the Fourier kernel $\Phi_{d}(k y)$ [it possesses the Taylor expansion (A11) around the origin] is the reason for the analyticity of the velocity correlator that passively advected fields "feel" at small scales in the Batchelor regime. 


\section{A.2. Correlation Functions of Solenoidal and Potential Fields}

Two important special cases of the field $u^{i}$ are incompressible (solenoidal) and irrotational (potential) fields. In the Fourier space, we have

$$
\begin{aligned}
& \tilde{\kappa}(k)=-\kappa(k) \quad \text { (solenoidal) } \\
& \kappa(k)=0 \quad \text { (potential). }
\end{aligned}
$$

In the $\mathbf{x}$ space, solenoidality and potentiality impose differential relations between the longitudinal and the transverse correlation functions. Namely,

$$
\begin{array}{rlr}
\kappa_{N N}(y) & =y \kappa_{L L}^{\prime}(y)+(d-1) \kappa_{L L}(y) & \text { (solenoidal) }, \\
\kappa_{L L}(y) & =\frac{1}{d-1}\left[y \kappa_{N N}^{\prime}(y)+\kappa_{N N}(y)\right] \quad \text { (potential). }
\end{array}
$$

[The relation (A20) is known as the von Kármán condition.] The equivalence of the above relations in their $\mathbf{k}$ and $\mathbf{x}$ space forms can, of course, be easily demonstrated with the aid of the formulas (A14) and (A15).

We may summarize the properties of the solenoidal fields as follows

$$
\begin{aligned}
\kappa^{i j}(\mathbf{k}) & =\frac{1}{d-1}\left(\delta^{i j}-\frac{k^{i} k^{j}}{k^{2}}\right) I(k), \\
\kappa^{i j}(\mathbf{y}) & =\kappa_{L L}(y) \delta^{i j}+\frac{1}{d-1} y \kappa_{L L}^{\prime}(y)\left(\delta^{i j}-\frac{y^{i} y^{j}}{y^{2}}\right),
\end{aligned}
$$

where, from (A14) and (A17),

$$
\begin{aligned}
\kappa_{L L}(y) & =\frac{1}{d} \frac{S_{d}}{(2 \pi)^{d}} \int_{0}^{\infty} \mathrm{d} k k^{d-1} \Phi_{d+2}(k y) I(k), \\
I(y) & =\frac{1}{y^{d-1}} \frac{\partial}{\partial y} y^{d} \kappa_{L L}(y)=\frac{S_{d}}{(2 \pi)^{d}} \int_{0}^{\infty} \mathrm{d} k k^{d-1} \Phi_{d}(k y) I(k) .
\end{aligned}
$$

These formulas are especially important because they must always hold for the correlation functions of the magnetic field, which is solenoidal. For example, the formula (A24) imposes a nontrivial (and somewhat different from that for the energy) constraint on the class of functions eligible to be longitudinal correlation functions of a solenoidal field $u^{i}(\mathbf{x})$.

Note that, if $\kappa_{L L}(y)$ decays at large scales $(y \rightarrow \infty)$ faster than $1 / y^{d}$, then the formula (A25) implies an often-used property of the energy function

$$
\int_{0}^{\infty} \mathrm{d} y y^{d-1} I(y)=0 .
$$

This means that $I(y)$ cannot remain positive for all $y$, and that $I(k)$, which is the inverse Fourier transform of $I(y)$, vanishes at $k=0$. In view of the expansion properties of $\Phi_{d}(k y)$ [see (A11)], the latter implies that the spectrum of a solenoidal field has the following scaling in the limit of small wave numbers:

$$
k^{d-1} I(k) \sim k^{d+1} \quad \text { as } \quad k \rightarrow 0 .
$$

In 3D, this implies the well-known $k^{4}$ infrared scaling of the spectra of solenoidal fields (Monin and Yaglom 1975). 


\section{A.3. The Realizability Inequalities}

It is easy to see that the formula (A5), which is a consequence of the homogeneity of space, can be recast in the following form:

$$
\kappa^{i j}(\mathbf{k})=L^{-d}\left\langle u^{i}(\mathbf{k}) u^{j *}(\mathbf{k})\right\rangle,
$$

where $L^{d}$ is the volume of space (henceforth set to 1) and the star means complex conjugation. Let us pick an arbitrary real unit vector $\mathbf{n}$ and take a full convolution of $n_{i} n_{j}$ with the above expression:

$$
n_{i} n_{j} \kappa^{i j}(\mathbf{k})=\left\langle|\mathbf{n} \cdot \mathbf{u}|^{2}\right\rangle
$$

whence, and by using (A6), we obtain, for all $\mathbf{k}$,

$$
\begin{gathered}
0 \leq n_{i} n_{j} \kappa^{i j}(\mathbf{k}) \leq\left\langle|\mathbf{u}|^{2}\right\rangle=\kappa^{i i}(\mathbf{k}), \\
0 \leq \kappa(k)+\tilde{\kappa}(k) \frac{(\mathbf{n} \cdot \mathbf{k})^{2}}{k^{2}} \leq d \kappa(k)+\tilde{\kappa}(k) .
\end{gathered}
$$

We may now write $(\mathbf{n} \cdot \mathbf{k})^{2}=k^{2} \cos ^{2} \theta$, where $\theta$ is the (arbitrary) angle between $\mathbf{k}$ and $\mathbf{n}$. Thus we arrive at the following realizability theorem: for any value of $\theta$ and any $k$ the following two inequalities must hold:

$$
\begin{gathered}
\kappa(k)+\tilde{\kappa}(k) \cos ^{2} \theta \geq 0, \\
(d-1) \kappa(k)+\tilde{\kappa}(k) \sin ^{2} \theta \geq 0 .
\end{gathered}
$$

In practice, we will be mostly content to use just the particular cases of the above arising from setting $\theta=0$ and $\theta=\pi / 2$ :

$$
\begin{gathered}
\kappa(k)+\tilde{\kappa}(k) \geq 0, \\
\kappa(k) \geq 0 .
\end{gathered}
$$

Let us now derive the realizability constraints that operate in the configuration space. Consider the two-point structure function of the field $u^{i}$ :

$$
\left\langle\left[u^{i}(\mathbf{y})-u^{i}(0)\right]\left[u^{j}(\mathbf{y})-u^{j}(0)\right]\right\rangle=2 \kappa^{i j}(0)-2 \kappa^{i j}(\mathbf{y}),
$$

take the full double dot product of the above expression with an arbitrary unit vector $\mathbf{n}$, and use the expression (A4) for the correlation tensor $\kappa^{i j}$. The resulting realizability theorem is

$$
\kappa_{L L}(0)-\kappa_{L L}(y) \cos ^{2} \theta-\frac{\kappa_{N N}(y)}{d-1} \sin ^{2} \theta=\frac{1}{2}\left\langle\left|\mathbf{n} \cdot\left[u^{i}(\mathbf{y})-u^{i}(0)\right]\right|^{2}\right\rangle \geq 0
$$

for any $y$ and any $\theta$ (the angle between $\mathbf{n}$ and $\mathbf{y}$ ). The inequalities of most practical value are again obtained for $\theta=0$ and $\theta=\pi / 2$ :

$$
\begin{gathered}
\kappa_{L L}(y) \leq \kappa_{L L}(0), \\
\kappa_{N N}(y) \leq(d-1) \kappa_{L L}(0)=\kappa_{N N}(0) .
\end{gathered}
$$

Thus, the two-point correlation functions can never exceed the values they take when the two points coincide. 


\section{A.4. Small-Scale Expansion of Second-Order Correlation Functions}

Since much of this work is concerned with one-point statistics, let us establish a correspondence between one- and two-point correlation properties of our field $u^{i}$. For $y \rightarrow 0$, we will routinely expand

$$
\kappa^{i j}(\mathbf{y})=\kappa_{0} \delta^{i j}-\frac{1}{2} \kappa_{2}\left(y^{2} \delta^{i j}+2 a y^{i} y^{j}\right)+\frac{1}{4} \kappa_{4} y^{2}\left(y^{2} \delta^{i j}+2 b y^{i} y^{j}\right)+\cdots .
$$

Here $a$ and $b$ are the so-called compressibility parameters which change within the intervals

$$
-\frac{1}{d+1} \leq a \leq 1 \text { and }-\frac{2}{d+3} \leq b \leq 2,
$$

where the lower bounds correspond to the incompressible case, the upper bounds to the irrotational one. Making use of the formula (A11), we expand the expressions (A14) and (A15) for $\kappa_{L L}(y)$ and $\kappa_{N N}(y)$ at

small $y$, substitute them into (A4), and, by comparing with (A40), establish the small-scale expansions of the velocity correlation functions in the $\mathbf{x}$ space,

$$
\begin{aligned}
\kappa_{L L}(y) & =\kappa_{0}-\frac{1}{2}(1+2 a) \kappa_{2} y^{2}+\frac{1}{4}(1+2 b) \kappa_{4} y^{4}+\cdots, \\
\kappa_{N N}(y) & =(d-1)\left(\kappa_{0}-\frac{1}{2} \kappa_{2} y^{2}+\frac{1}{4} \kappa_{4} y^{4}+\cdots\right),
\end{aligned}
$$

and the expressions for the parameters of the correlator (A40) in terms of the spectral characteristics of the velocity field,

$$
\begin{aligned}
\kappa_{0} & =\frac{1}{d} \int \frac{\mathrm{d}^{d} k}{(2 \pi)^{d}} I(k), \\
\kappa_{2} & =\frac{1}{d(d+2)} \int \frac{\mathrm{d}^{d} k}{(2 \pi)^{d}} k^{2}[(d+2) \kappa(k)+\tilde{\kappa}(k)], \\
\kappa_{4} & =\frac{1}{2 d(d+2)(d+4)} \int \frac{\mathrm{d}^{d} k}{(2 \pi)^{d}} k^{4}[(d+4) \kappa(k)+\tilde{\kappa}(k)], \\
a & =\kappa_{2}^{-1} \frac{1}{d(d+2)} \int \frac{\mathrm{d}^{d} k}{(2 \pi)^{d}} k^{2} \tilde{\kappa}(k), \\
b & =\kappa_{4}^{-1} \frac{1}{d(d+2)(d+4)} \int \frac{\mathrm{d}^{d} k}{(2 \pi)^{d}} k^{4} \tilde{\kappa}(k),
\end{aligned}
$$

where the $\mathbf{k}$-space integrations of radial functions could, of course, have been written more explicitly as

$$
\int \frac{\mathrm{d}^{d} k}{(2 \pi)^{d}}=\frac{S_{d}}{(2 \pi)^{d}} \int_{0}^{\infty} \mathrm{d} k k^{d-1}
$$

Finally, let us remark that the expression (14) that was obtained in Sec. 2 for the initial growth rate of the total magnetic energy can be, in view of the above, written as follows:

$$
\gamma=\frac{1}{2}(d-1)(d a+2) \kappa_{2}=\frac{1}{2} \frac{d-1}{d+1}(d+2+\beta) \kappa_{2},
$$

where $\beta=d[1+a(d+1)]$. The above expression is naturally in agreement with the results known in the one-point setting (see, e.g., Boldyrev and Schekochihin 2000; Schekochihin and Kulsrud 2001). Another convenient expression (or definition) for $\gamma$, that we use in Sec. 3, is

$$
\gamma=\frac{1}{2 A}(1+2 a) \kappa_{2}=\frac{\left|\kappa_{L L}^{\prime \prime}(0)\right|}{2 A},
$$

where $A$ is defined by the formula (22). 


\section{A.5. Angle-Integration Identities}

Dealing with $d$-dimensional wave-number integrations while deriving the mode-coupling equation (11), the evolution laws (13) and (16), and the SSF equation (21), required repeated use of some simple angleintegration identities which we list here for completeness:

$$
\begin{aligned}
\int \mathrm{d} \Omega_{d} & =S_{d}=\frac{2 \pi^{d / 2}}{\Gamma(d / 2)}, \\
\int \mathrm{d} \Omega_{d} n_{i} n_{j} & =\frac{S_{d}}{d} \delta_{i j}, \\
\int \mathrm{d} \Omega_{d} n_{i} n_{j} n_{k} n_{l} & =\frac{S_{d}}{d(d+2)}\left(\delta_{i j} \delta_{k l}+\delta_{i k} \delta_{j l}+\delta_{i l} \delta_{k j}\right), \\
\iint \mathrm{d} \Omega_{d} \mathrm{~d} \Omega_{d}^{\prime}\left(\mathbf{n} \cdot \mathbf{n}^{\prime}\right)^{2} & =\frac{S_{d}^{2}}{d} \\
\iint \mathrm{d} \Omega_{d} \mathrm{~d} \Omega_{d}^{\prime}\left(\mathbf{n} \cdot \mathbf{n}^{\prime}\right)^{4} & =\frac{3 S_{d}^{2}}{d(d+2)},
\end{aligned}
$$

where $\mathrm{d} \Omega_{d}=\mathrm{d} \varphi \sin \theta_{1} \mathrm{~d} \theta_{1} \ldots \sin ^{d-2} \theta_{d-2} \mathrm{~d} \theta_{d-2}$ is the $d$-dimensional angular differential, and $\mathbf{n}=\mathbf{k} / k$ is a unit vector in the direction of $\mathbf{k}$. The above identities can be derived in various ways, one of them being via formula (A9).

\section{B. THE FURUTSU-NOVIKOV FORMULA}

Here we state an extremely useful Gaussian-averaging theorem that is widely used throughout this work. This result is due to Furutsu (1963) and Novikov (1965) and is generally referred to as the Furutsu-Novikov, or "Gaussian-integration," formula.

Consider a random Gaussian vector field $u^{i}(q)$, where $q$ is the aggregate of all variables it depends on (such as time, spatial position, Fourier variable, or vector index). Let $R[\mathbf{u}]$ be some functional of the field $u^{i}$. Then the following holds:

$$
\left\langle u^{i}(q) R[\mathbf{u}]\right\rangle=\int \mathrm{d} q^{\prime}\left\langle u^{i}(q) u^{j}\left(q^{\prime}\right)\right\rangle\left\langle\frac{\delta R[\mathbf{u}]}{\delta u^{j}\left(q^{\prime}\right)}\right\rangle
$$

where the integration is over all possible values of $q^{\prime}$.

This property of Gaussian fields is simply a generalization of the well-known "splitting" property of the Gaussian averages.

\section{LARGE-SCALE CORRECTIONS TO THE INERTIAL-RANGE ASYMPTOTICS}

In this Appendix, we work out the first "large-scale" corrections to the inertial-range asymptotics (77) and (78) of the Kazantsev particle's mass and the potential it lives in, as well as the appropriate corrected asymptotic forms of the solutions. These corrections are needed in order for us to get a better idea of the general form of the Kazantsev quantum mechanics. They were also appealed to when the asymptotic matching was performed in $\S 3.3$. 
In order to obtain the desired corrections, we must use the expansion (A40) of the velocity correlator (50) up to the fourth order in $y$. The fourth-order Taylor expansions of the renormalized diffusivities are

$$
\begin{aligned}
& K(y) \simeq 2 \eta+\frac{1}{2}(1+2 a) \kappa_{2} y^{2}-\frac{1}{4}(1+2 b) \kappa_{4} y^{4}, \\
& Q(y) \simeq 2 \eta+\frac{1}{2} \kappa_{2} y^{2}-\frac{1}{4} \kappa_{4} y^{4},
\end{aligned}
$$

and we find the following corrected asymptotics for mass and potential in the inertial range:

$$
\begin{aligned}
m(y) \simeq & \frac{1}{(1+2 a) \kappa_{2} y^{2}}\left[1+\frac{1}{2} \frac{1+2 b}{1+2 a} \frac{\kappa_{4}}{\kappa_{2}} y^{2}\right], \\
V(y) \simeq & -V_{0}+V_{2} y^{2}, \\
& \quad \text { where } \quad V_{2}=\frac{3}{8}\left[3 d-7-4 b-(d-3)^{2} \frac{1+4 a-2 b}{6(1+2 a)^{2}}\right] \kappa_{4} .
\end{aligned}
$$

The correction $V_{2} y^{2}$ in the formula (C4) is positive in the $3 \mathrm{D}$ incompressible case, changes sign at $b=1 / 2$, and remains negative for all $b>1 / 2$, including in the irrotational case. This means that the value $V(y) \simeq-V_{0}$ represents an intermediate "plateau," and not the bottom of the potential well, which extends further down [how far down is, of course, impossible to determine on the basis of the Taylor expansion (C4); a representative example of such a potential is given in Fig. 9]. The danger thus arises that the Kazantsev particle might slide into this deeper recess and thereby destroy the small-scale universality, upon which hinged the validity of the results obtained in $\S 3.3$. Whether or not this happens in reality may depend on the large-scale structure of the potential and on the interplay between it and the structure of the variable mass $m(y)$. The importance of the latter effect is clear from the form of the eigenvalue problem (64) itself, which can be reinterpreted as a Schrödinger equation for a particle with constant mass and zero energy in a $\lambda$-dependent effective potential $U_{\lambda}(y)$ :

$$
\begin{gathered}
-\psi^{\prime \prime}+U_{\lambda}(y) \psi=0, \\
U_{\lambda}(y)=2 m(y)[V(y)+\lambda \gamma] .
\end{gathered}
$$

We must now treat $\lambda$ as a parameter to be chosen in such way that the zero-energy eigenstate exist and be a ground state. The effective potential $U_{\lambda}(y)$ explicitly incorporates both influences [variable mass and potential $V(y)$ ] that act on the Kazantsev particle. In the inertial range, it is, of course, an inverse-square potential. Due to the inverse-square decay of $m(y)$, the fourth-order effects lead only to a constant correction, namely,

$$
\begin{aligned}
U_{\lambda}(y) \simeq-\frac{\lambda_{\max }-\lambda}{A y^{2}}+U_{2}, \\
\quad \text { where } U_{2}=\frac{1}{\gamma A}\left[V_{2}-\frac{1}{4}(1+2 b) \kappa_{4} \frac{\lambda_{\max }-\lambda}{A}\right] .
\end{aligned}
$$

The solutions of Eq. (C5) with the effective potential (C7) are the modified Bessel functions:

$$
\psi(y)=y^{1 / 2}\left[c_{1} I_{\sqrt{\left(\lambda-\lambda_{0}\right) / A}}\left(U_{2}^{1 / 2} y\right)+c_{2} K_{\sqrt{\left(\lambda-\lambda_{0}\right) / A}}\left(U_{2}^{1 / 2} y\right)\right] .
$$

These are the solutions we referred to in justifying the asymptotic (91) of $\S 3.3$.

The potentials $V(y)$ and $U_{\lambda}(y)$ for a particular 3D irrotational velocity field with the transverse correlation function chosen to be $\kappa_{N N}(y)=2 \kappa_{0} \exp \left(-\kappa_{2} y^{2} / 2\right)$, are shown in Fig. 9 and Fig. 10. In this specific case, 
the straightforward numerical solution of the eigenvalue problem (64) proved that the Kazantsev particle was "heavy enough" to settle on the flat "porch" corresponding to the inertial-range asymptotic $V(y) \simeq-V_{0}$, rather than slide off towards larger scales and deeper into the potential well. The small-scale universality therefore persisted.

\section{REFERENCES}

Alfvén, H. 1937a, Arkiv. Mat. Astron. Fysik, 25B, No. 29

Alfvén, H. 1937b, Z. Physik, 107, 579

Artamonova, O. V. and Sokoloff, D. D. 1986, Vestn. Mosk. Univ. Ser. 3. Fiz. Astron., 27, No. 3, 8

Batchelor, G. K. 1950, Proc. R. Soc. London, Ser. A, 201, 405

Batchelor, G. K. 1959, J. Fluid. Mech., 5, 113

Beck, R., Brandenburg, A., Moss, D., Shukurov, A, and Sokoloff, D. 1996, ARA\&A, 34, 155

Bernard, D., Gawędzki, K., and Kupiainen, A. 1998, J. Stat. Phys., 90, 519

Biermann, L. 1950, Z. Naturforsch., 5a, 65

Biermann, L. and Schlüter, A. 1951, Phys. Rev., 82, 863

Blackman, E. G. 1998, ApJ, 496, L17

Blackman, E. G. and Field, G. B. 1999, ApJ, 521, 597

Blackman, E. G. and Field, G. B. 2000, ApJ, 534, 984

Boldyrev, S. A. 2000, preprint (astro-ph/0006267)

Boldyrev, S. A. 2001, ApJ, 562, 1081

Boldyrev, S. A. and Schekochihin, A. A. 1998, unpublished

Boldyrev, S. A. and Schekochihin, A. A. 2000, Phys. Rev. E, 62, 545

Braginskii, S. I. 1965a, Rev. Plasma Phys., 1, 205

Braginskii, S. I. 1965b, Sov. Phys. JETP, 20, 726

Brandenburg, A. 2001, ApJ, 550, 824

Brandenburg, A. and Subramanian, K. 2000, A\&A, 361, L33

Cattaneo, F. 1997, in: SCORe '96: Solar Convection and Oscillations and their Relationship, ed. F. P. Pijpers et al. (Dordrecht: Kluwer), 201

Cattaneo, F. 1999, in: Motions in the Solar Atmosphere, ed. A. Hanslmeier and M. Messerotti (Dordrecht: Kluwer), 119

Cattaneo, F. and Hughes, D. W. 1996, Phys. Rev. E, 54, R4532 
Chandran, B. D. G. 1998, ApJ, 492, 179

Chertkov, M., Falkovich, G., Kolokolov, I., and Vergassola, M. 1999, Phys. Rev. E, 83, 4065

Childress, S. and Gilbert, A. 1995, Stretch, Twist, Fold: The Fast Dynamo (Berlin: Springer)

Chou, H. 2001a, ApJ, 552, 803

Chou, H. 2001b, ApJ, 556, 1038

Descartes, R. 1637, "Discours de la méthode pour bien conduire sa raison, et chercher la verité dans les sciences," in: CEuvres de Descartes, Tome I (Paris: Victor Cousin, 1824)

Erdélyi, A. (ed.) 1954, Tables of Integral Transforms, Volume II (New York: McGraw-Hill)

Fermi, E. 1949, Phys. Rev., 75, 1169

Field, G. B., Blackman, E. G., and Chou, H. 1999, ApJ, 513, 638

Field, G. B. and Blackman, E. G. 2001, preprint (astro-ph/0111470)

Furutsu, K. 1963, J. Res. NBS, 67D, 303

Goldreich, P. and Sridhar, S. 1995, ApJ, 438, 763

Goldreich, P. and Sridhar, S. 1997, ApJ, 485, 680

Gruzinov, A., Cowley, S., and Sudan, R. 1996, Phys. Rev. Lett., 77, 4342

Gruzinov, A. V. and Diamond, P. H. 1994, Phys. Rev. Lett., 72, 1651

Gruzinov, A. V. and Diamond, P. H. 1995, Phys. Plasmas, 2, 1941

Gruzinov, A. V. and Diamond, P. H. 1996, Phys. Plasmas, 3, 1853

Hall, J. S. and Mikesell, A. M. 1949, AJ, 54, 187

Hiltner, W. A. 1949, ApJ, 109, 471

Howard, A. M. and Kulsrud, R. M. 1997, ApJ, 483, 648

Iroshnikov, P. 1964, Sov. Astron, 7, 566

van Kampen, N. G., 1976, Phys. Rep., 24, 171

Kazantsev, A. P. 1968, Sov. Phys. JETP, 26, 1031

Kichatinov, L. L. 1985, Magnetohydrodynamics, 21, 1

Kinney, R. M., Chandran, B., Cowley, S., and McWilliams, J. C. 2000, ApJ, 545, 907

Kinney, R. M, Cowley, S., McWilliams, J. C., and Opher, M. 2000, unpublished

Kleeorin, N. I., Ruzmaikin, A. A., and Sokoloff, D. D. 1986, Plasma Astrophysics, ESA SP-251, 557

Kraichnan, R. H. 1965, Phys. Fluids, 8, 1385 
Kraichnan, R. H. 1968, Phys. Fluids, 11, 945

Kraichnan, R. H. 1994, Phys. Rev. Lett., 72, 1016

Kraichnan, R. H. and Nagarajan, S. 1967, Phys. Fluids, 10, 859

Kronberg, P. P. 1994, Rep. Prog. Phys., 57, 325

Kulsrud, R. M. 1999, ARA\&A, 37, 37

Kulsrud, R. M. 2000, in: Proceedings of the International School of Physics "Enrico Fermi," Course CXLII, ed. B. Coppi, A. Ferrari, and E. Sindoni (Amsterdam: IOS Press), 107

Kulsrud, R. M. and Anderson, S. W. 1992, ApJ, 396, 606

Kulsrud, R. M., Cen, R., Ostriker, J. P., and Ryu, D. 1997a, ApJ, 480, 481

Kulsrud, R. M., Cowley, S. C., Gruzinov, A. V., and Sudan, R. N. 1997b, Phys. Rep., 283, 213

Landau, L. D. and Lifshitz, E. M. 1977, Quantum Mechanics (Oxford: Pergamon)

Malyshkin, L. 2001, Ph. D. Thesis, Princeton University; preprint (astro-ph/0110081)

Maron, J. and Blackman, E. G. 2001, preprint (astro-ph/0110018); ApJ, submitted

Maron, J., Cowley, S., and McWilliams, J. 2002, preprint (astro-ph/0111008); ApJ, submitted

Maslova, T. B. and Ruzmaikin, A. A. 1987, Magnetohydrodynamics, 23, 231

Meneguzzi, M., Frisch, U., and Pouquet, A. 1981, Phys. Rev. Lett., 47, 1060

Moffatt, H. K. 1978, Magnetic Field Generation in Electrically Conducting Fluids (Cambridge: Cambridge University Press)

Molchanov, S. A., Ruzmaikin, A. A., and Sokoloff, D. D. 1983, Magnetohydrodynamics, 19, 402

Monin, A. S. and Yaglom, A. M. 1975, Statistical Fluid Mechanics: Mechanics of Turbulence, Volume II (Cambridge, Mass.: The MIT Press)

Morse P. M., and Feshbach, H. 1953, Methods of Theoretical Physics (New York: McGrow Hill)

Novikov, E. A. 1965, Sov. Phys. JETP, 20, 1290

Novikov, V. G., Ruzmaikin, A. A., and Sokoloff, D. D. 1983, Sov. Phys. JETP, 58, 527

Ott, E. 1998, Phys. Plasmas, 5, 1636

Parker, E. N. 1955, ApJ, 122, 293

Parker, E. N. 1979, Cosmical Magnetic Fields: Their Origin and Their Activity (Oxford: Clarendon)

Rafikov, R. R. and Kulsrud, R. M. 2000, MNRAS, 314, 839

Rogachevskii, I. and Kleeorin, N. 1997, Phys. Rev. E, 56, 417

Ruzmaikin, A. A., Shukurov, A. M., and Sokoloff, D. D. 1988, Magnetic Fields of Galaxies (Dordrecht: Kluwer) 
Ruzmaikin, A. A. and Sokoloff, D. D. 1981, Sov. Astron. Lett., 7, 388

Sakharov, A. D. 1982, Selected Scientific Works, (New York: Marcel Dekker), Divertissment 11

Schekochihin, A., Cowley, S., Maron, J., and Malyshkin, L. 2002a, Phys. Rev. E, 65, 016305

Schekochihin, A. A. and Kulsrud, R. M. 1998, unpublished

Schekochihin, A. A. and Kulsrud, R. M. 2001, Phys. Plasmas, 8, 4937

Schekochihin, A. A., Maron, J. L., Cowley, S. C., and McWilliams, J. C. 2002b, ApJ, submitted

Steenbeck, M., Krause, F., und Rädler, K.-H. 1966, Z. Naturforsch., 21a, 369

Stein, E. M. 1993, Harmonic Analysis: Real-Variable Methods, Orthogonality, and Oscillatory Integrals (Princeton: Princeton University Press)

Subramanian, K. 1997, preprint (astro-ph/9708216)

Subramanian, K. 1998, MNRAS, 294, 718

Subramanian, K. 1999, Phys. Rev. Lett., 83, 2957

Vainshtein, S. I. 1970, Sov. Phys. JETP, 31, 87

Vainshtein, S. I. 1976, Sov. Phys. Usp., 19, 987

Vainshtein, S. I. 1980, Sov. Phys. JETP, 52, 1099

Vainshtein, S. I. 1982a, Magnetohydrodynamics, 18, 123

Vainshtein, S. I. 1982b, Sov. Phys. JETP, 56, 86

Vainshtein, S. I. and Cattaneo, F. 1992, ApJ, 393, 165

Vainshtein, S. I. and Kichatinov, L. L. 1986, J. Fluid Mech., 168, 73

Vainshtein, S. I. and Zeldovich, Ya. B. 1972, Sov. Phys. Usp., 15, 159

Vergassola, M. 1996, Phys. Rev. E, 53, R3021

Zeldovich, Ya. B. 1957, Sov. Phys. JETP, 4, 460

Zweibel, E. G. and Heiles, C. 1997, Nature, 385, 131

This preprint was prepared with the AAS LATEX macros v5.0. 


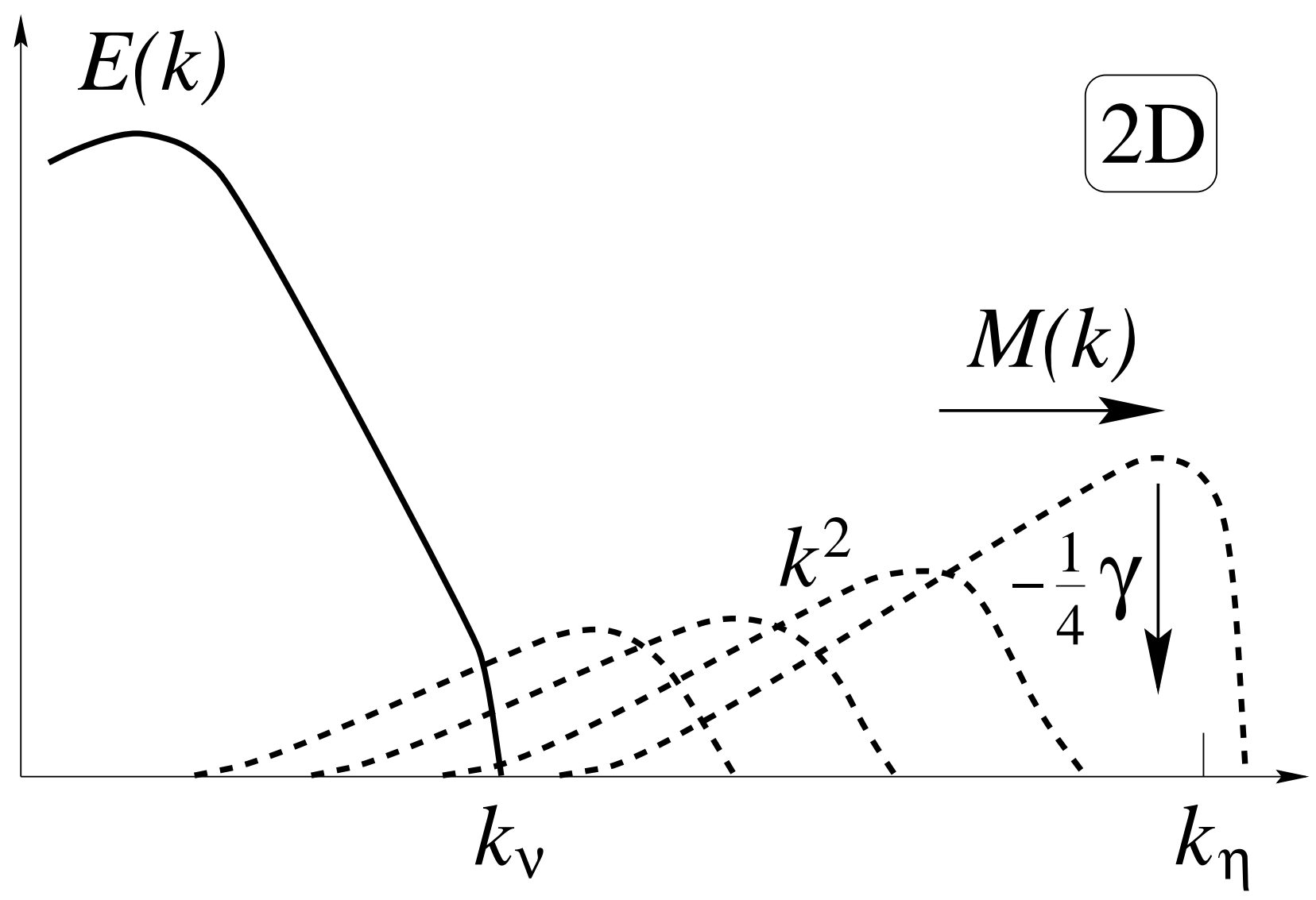

Fig. 1. - Sketch of the small-scale dynamo in 2D. The number of excited modes grows, while each individual mode decays. Both processes occur exponentially fast. The total growth rate of the magnetic energy in the diffusion-free regime is $2 \gamma$. The per-mode decay rate and the spectral index depend on the degree of compressibility. The numbers on the sketch correspond to the incompressible case. 


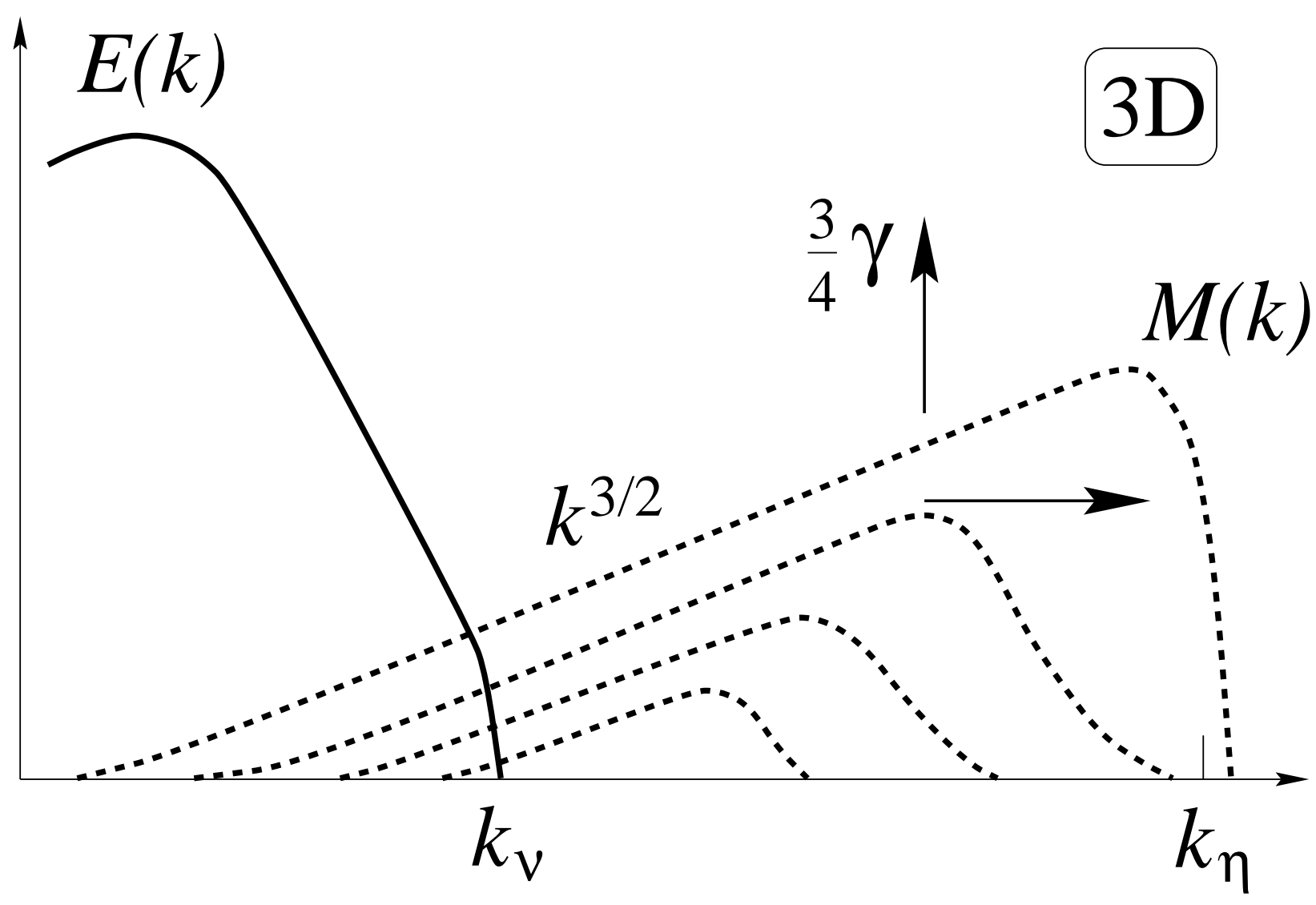

Fig. 2.- Sketch of the small-scale dynamo in 3D. Both the number of modes and the amplitudes of all individual modes grow exponentially in time. The total growth rate of the magnetic energy in the diffusionfree regime is $2 \gamma$. The per-mode growth rate depends on the degree of compressibility, but the spectrum is always $k^{3 / 2}$. The numbers on the sketch correspond to the incompressible case. 


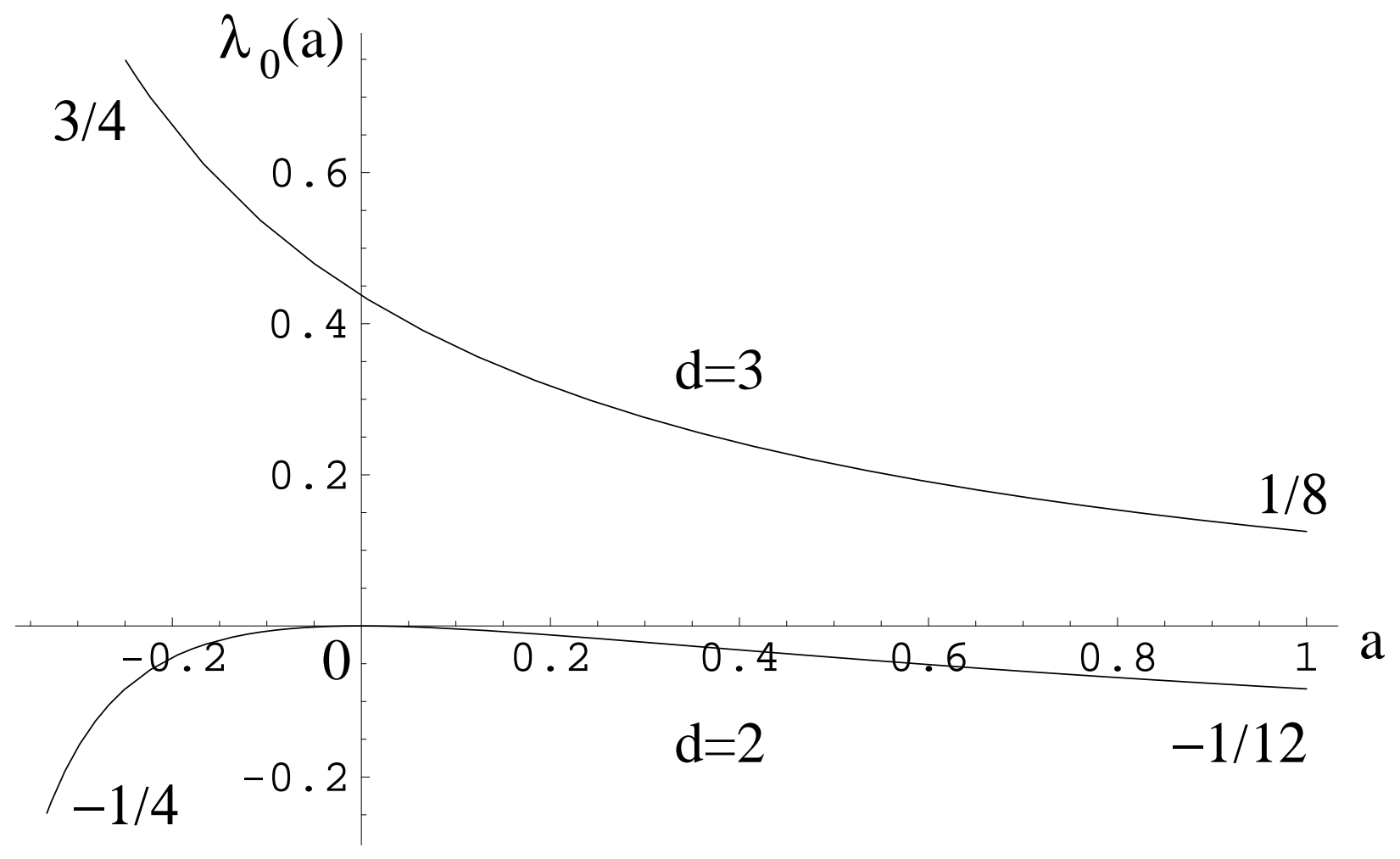

Fig. 3.- Dependence of the growth rate of each individual mode, $\lambda_{0}$, on the compressibility parameter $a$ in 2 and 3 dimensions [see formula (28)] and Table 2. 


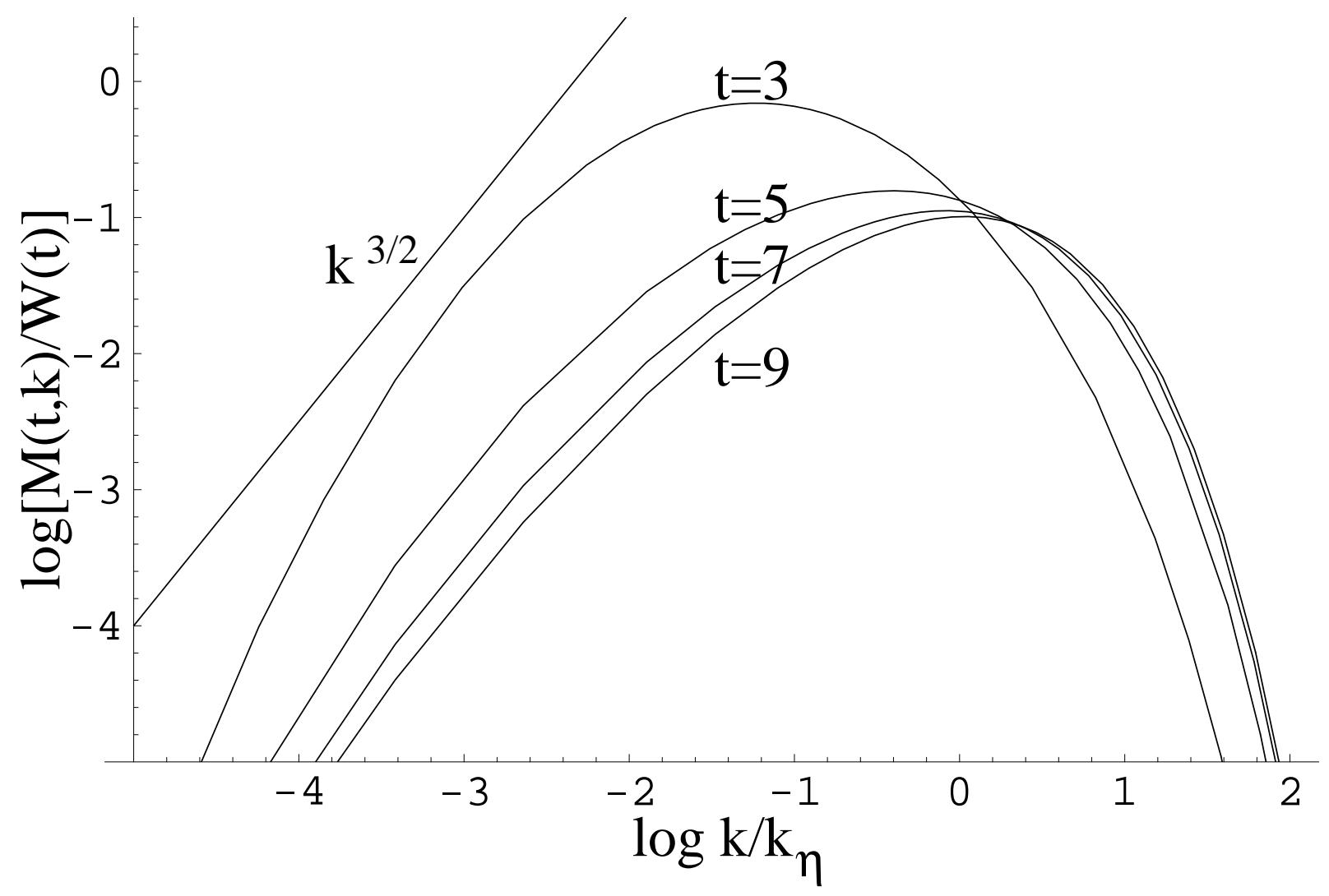

Fig. 4.- The log-log plot of the spectrum (35) in 3D incompressible flow. The spectrum $M(t, k)$ is normalized to the total energy $W(t)$ and shown at times $3 \gamma^{-1}, 5 \gamma^{-1}, 7 \gamma^{-1}$, and $9 \gamma^{-1}$. For reference, the line corresponding to the expected $k^{3 / 2}$ inertial-range spectrum is also plotted. The initial wave number was taken to be $k_{0}=0.05 k_{\eta}$. All wave numbers are normalized to the resistive wave number $k_{\eta}$. 


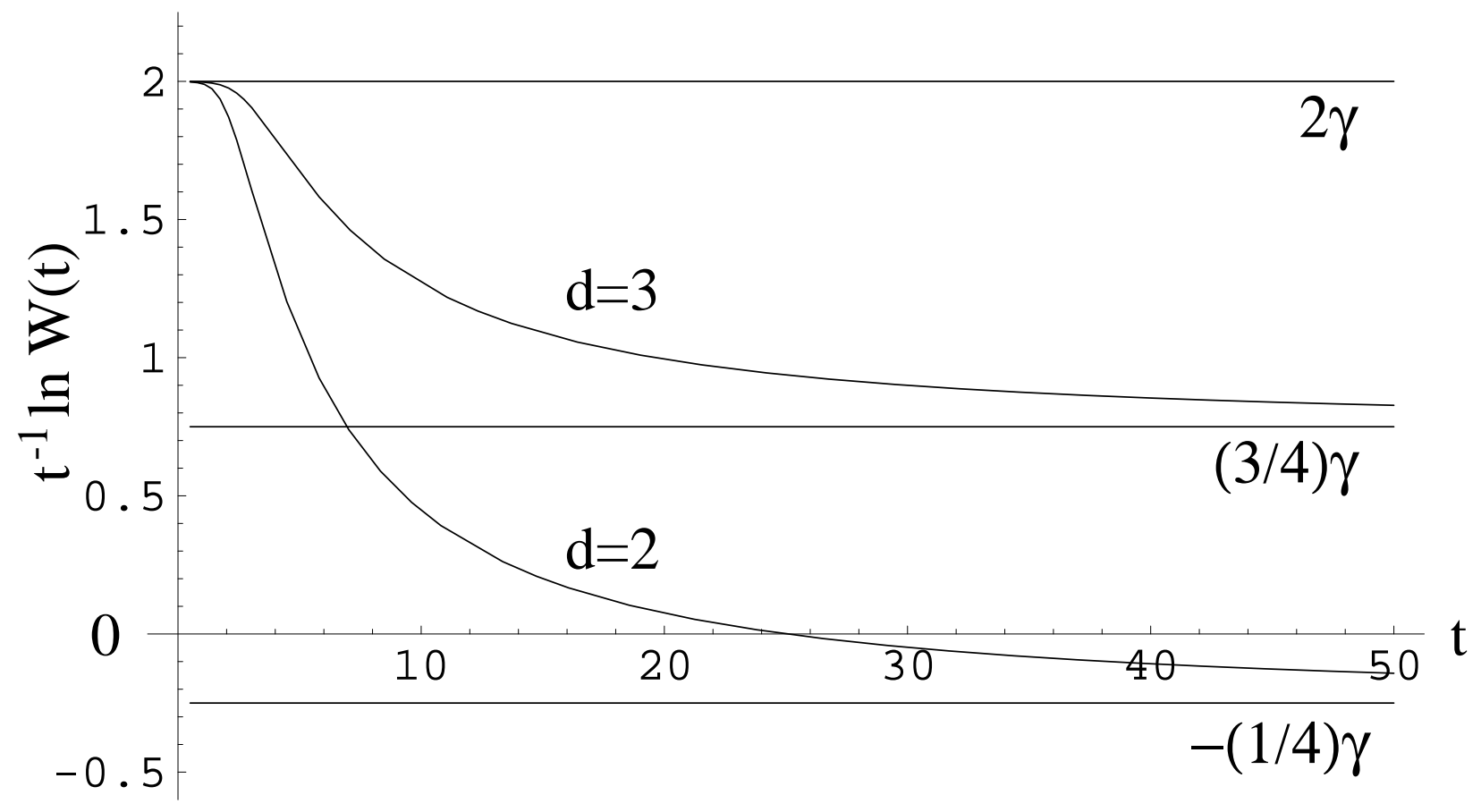

Fig. 5.- Evolution of the "effective growth rate" $\gamma_{\mathrm{eff}}(t)=t^{-1} \ln W(t)$ of the magnetic energy [see Eq. (36)] in two and three dimensions in the case of incompressible flow. The time is measured in units of $\gamma^{-1}$ [defined by the formula (14)]. 


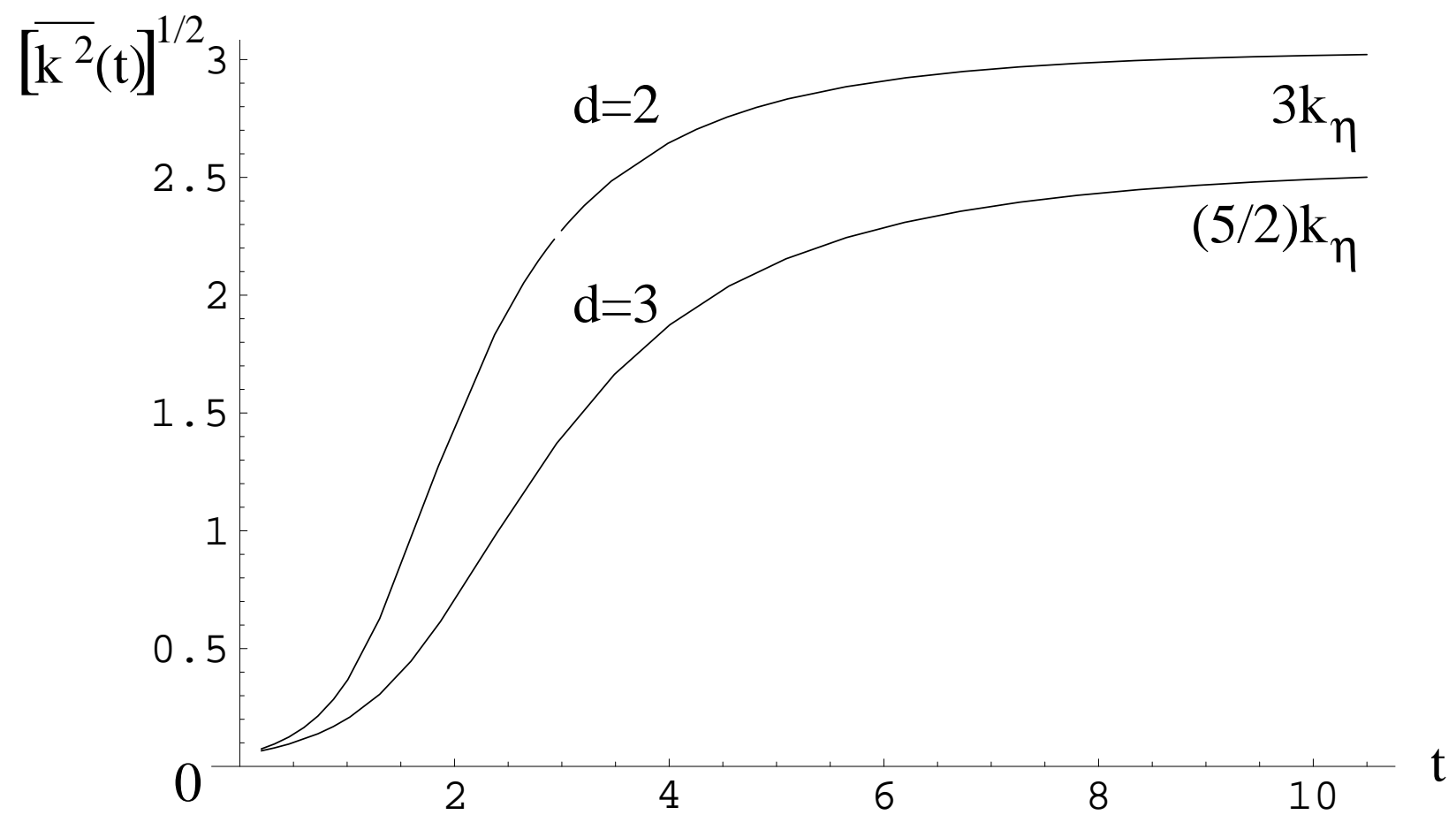

Fig. 6. Evolution of the mean square wave number of the magnetic fluctuations, $\left[\overline{k^{2}}(t)\right]^{1 / 2}$, in two and three dimensions in the case of incompressible flow. The time is measured in units of $\gamma^{-1}$ [defined by the formula (14)]. The wave numbers are measured in units of the inverse resistive scale $k_{\eta}$. The initial wave number was taken to be $k_{0}=0.05 k_{\eta}$. 


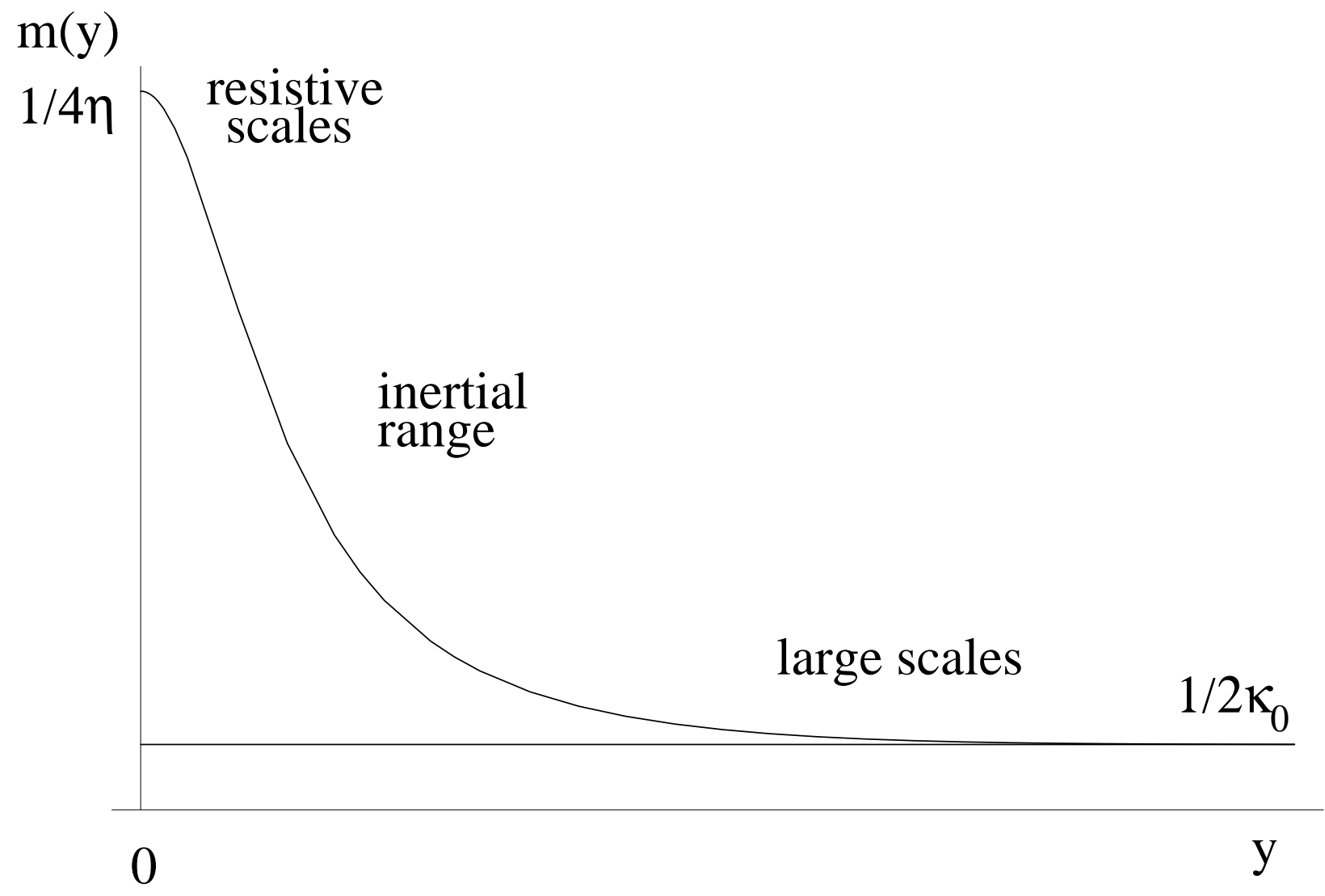

Fig. 7.- A schematic plot of the variable mass in Kazantsev quantum mechanics. 


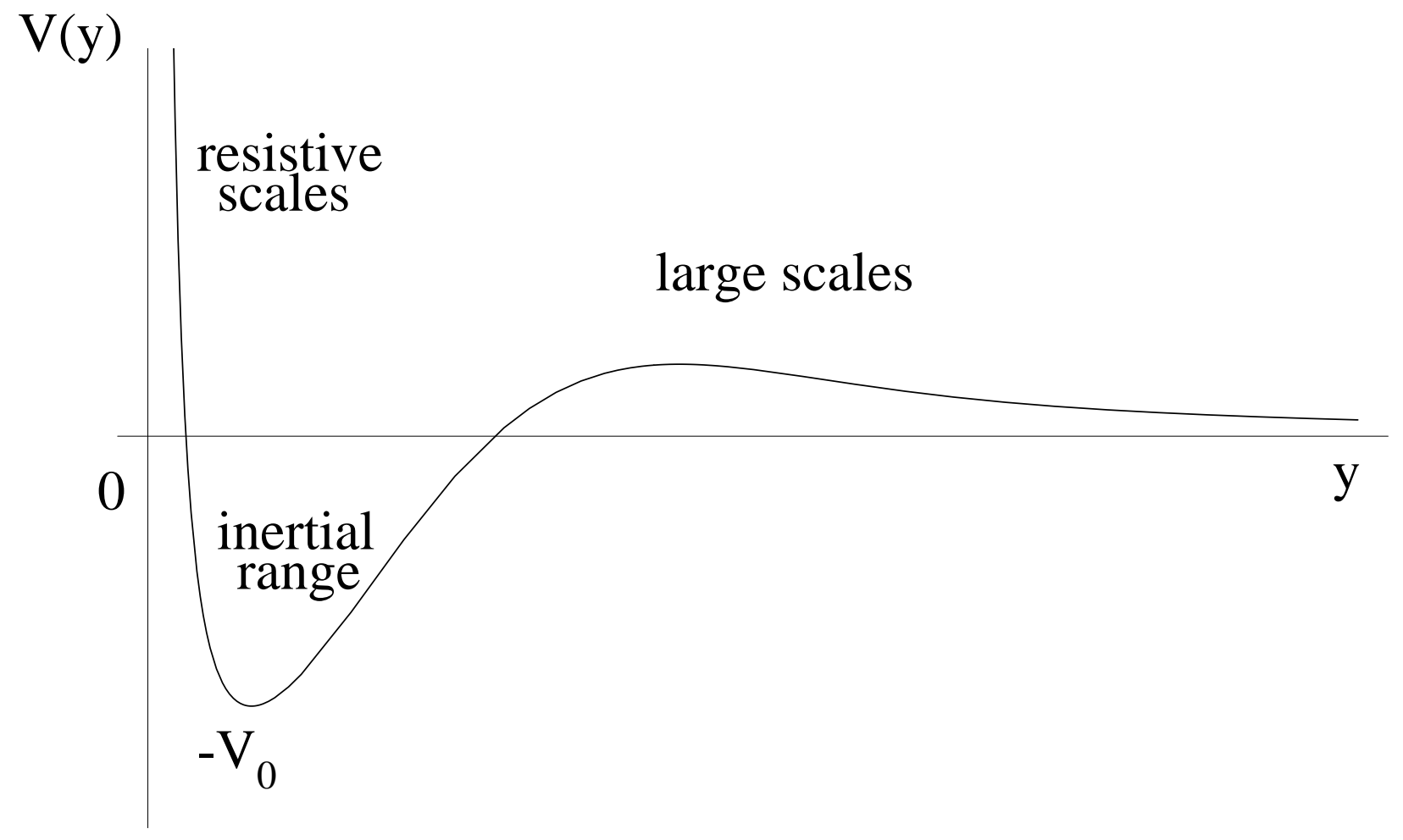

Fig. 8.- A schematic plot of the potential in Kazantsev quantum mechanics in three dimensions [see formula (62)]. In its particulars, this plot depicts the potential $V(y)$ for the 3D incompressible flow. 


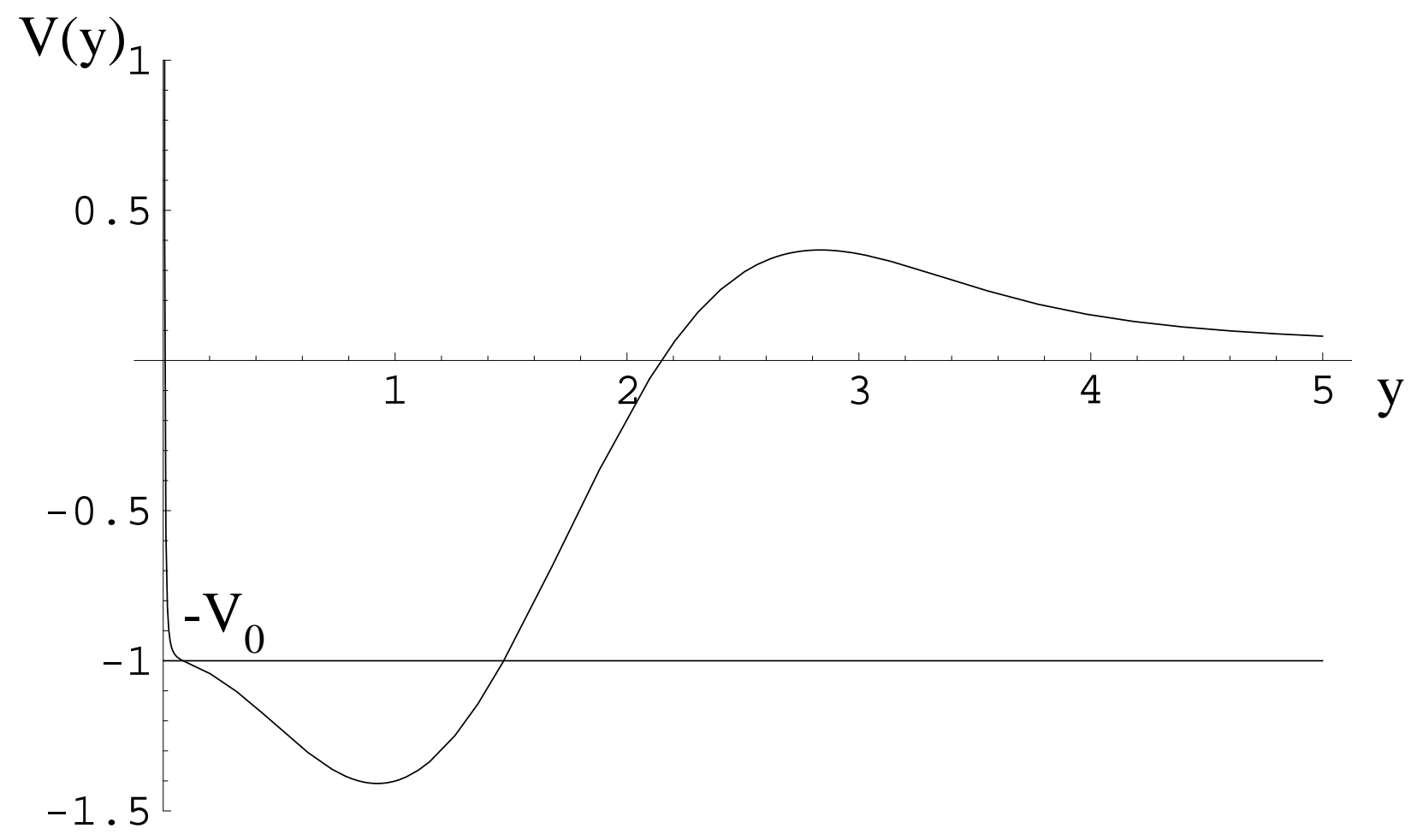

Fig. 9.- The Kazantsev potential in the case of 3D irrotational flow. For reference, the line corresponding to $V=-V_{0}$ is also shown. $V(y)$ is measured in units of $V_{0}, y$ in units of $\left(\kappa_{0} / \kappa_{2}\right)^{1 / 2}$. The transverse velocity correlation function was chosen in the form $\kappa_{N N}(y)=2 \kappa_{0} \exp \left(-\kappa_{2} y^{2} / 2\right)$. Due to the irrotational property of the flow, the longitudinal correlation function had to be calculated according to the formula (A21) of Appendix A.2. 


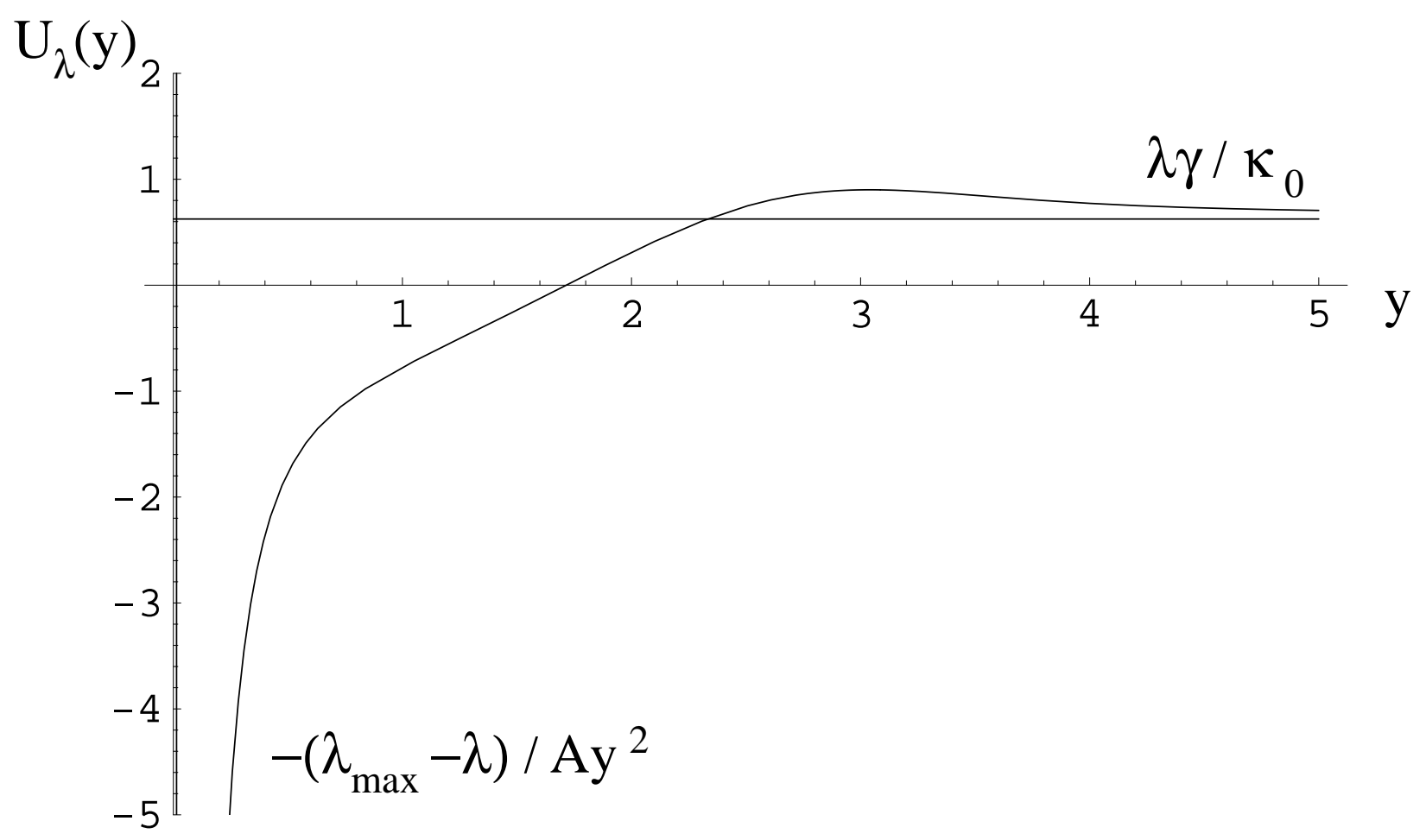

Fig. 10. - The form of the function $U_{\lambda}(y)=2 m(y)[V(y)+\lambda \gamma]$ which corresponds to the potential $V(y)$ plotted in Fig. 9 and $\lambda=\lambda_{0}=1 / 8$. The bottom of the potential well extends to $U_{\lambda} \sim-\kappa_{2} / \eta$ and is not shown. 
Table 1. Physical parameters in the Galaxy and Protogalaxy.

\begin{tabular}{|c|c|c|c|}
\hline Parameter & Notation & Galaxy (warm ISM) & Protogalaxy \\
\hline \multicolumn{4}{|c|}{ Densities and Temperatures } \\
\hline $\begin{array}{l}\text { neutral (hydrogen) density, } \mathrm{cm}^{-3} \\
\text { ion (proton) density, } \mathrm{cm}^{-3} \\
\text { temperature, } \mathrm{K}\end{array}$ & $\begin{array}{l}n_{n} \\
n_{i} \\
T \sim T_{i} \sim T_{e} \sim T_{n}\end{array}$ & $\begin{array}{l}1 \\
1 \\
10^{4}\end{array}$ & $\begin{array}{l}0 \\
10^{-3} \\
10^{6}\end{array}$ \\
\hline \multicolumn{4}{|c|}{ Length Scales, $\mathrm{cm}\left(3 \cdot 10^{18} \mathrm{~cm} \approx 1\right.$ parsec $)$} \\
\hline $\begin{array}{l}\text { system size } \\
\text { magnetic field coherence } \\
\text { energy-containing } \\
\text { viscous cutoff } \\
\text { neutral mean free path } \\
\text { ion mean free path } \\
\text { resistive cutoff } \\
\text { ion skin depth }\end{array}$ & $\begin{array}{l}- \\
\bar{L} \\
k_{\nu}^{-1} \sim \operatorname{Re}^{-3 / 4} L \\
\ell_{n} \sim v_{\mathrm{th}} \min \left\{\tau_{n n}, \tau_{n i}\right\} \\
\ell_{i} \sim v_{\mathrm{th}} \min \left\{\tau_{i i}, \tau_{i n}\right\} \\
k_{\eta}^{-1} \sim \operatorname{Pr}^{-1 / 2} k_{\nu}^{-1} \\
d_{i} \sim c / \omega_{p i}\end{array}$ & $\begin{array}{l}10^{22} \\
10^{21} \\
10^{20} \\
10^{16} \\
10^{14} \\
10^{12} \\
10^{9} \\
10^{7}\end{array}$ & $\begin{array}{l}10^{24} \\
\text { unknown } \\
10^{24} \\
10^{20} \\
- \\
10^{19} \\
10^{9} \\
10^{9}\end{array}$ \\
\hline \multicolumn{4}{|c|}{ Time Scales, $\sec \left(\pi \cdot 10^{7} \sec \approx 1\right.$ year $)$} \\
\hline $\begin{array}{l}\text { system lifetime } \\
\text { system rotation } \\
\text { largest-eddy turnover } \\
\text { viscous/smallest-eddy turnover } \\
\text { neutral-neutral collision } \\
\text { ion-neutral/neutral-ion collision } \\
\text { ion-ion collision }\end{array}$ & $\begin{array}{l}- \\
\bar{L} v_{\text {th }}^{-1} \\
\tau_{\text {eddy }} \sim\left(k_{\nu} u_{\text {eddy }}\right)^{-1} \\
\tau_{n n} \sim 4 \pi r_{\text {Bohr }}^{2} v_{\text {th }} n_{n} \\
\tau_{i n} \sim\left(m_{i} n_{i} / m_{n} n_{n}\right) \tau_{n i} \\
\tau_{i i} \sim\left(k_{B} T\right)^{3 / 2} m_{i}^{1 / 2}\left(e^{4} n_{i} \ln \Lambda\right)^{-1}\end{array}$ & $\begin{array}{l}10^{17} \\
10^{15} \\
10^{14} \\
10^{12} \\
10^{9} \\
10^{8} \\
10^{6}\end{array}$ & $\begin{array}{l}10^{17} \\
10^{17} \\
10^{14} \\
- \\
- \\
10^{12}\end{array}$ \\
\hline \multicolumn{4}{|c|}{ Velocities, $\mathrm{cm} / \mathrm{sec}$} \\
\hline $\begin{array}{l}\text { thermal/shock/largest-eddy } \\
\text { smallest-eddy }\end{array}$ & $\begin{array}{l}v_{\text {th }} \sim\left(k_{B} T / m_{i}\right)^{1 / 2} \\
u_{\text {eddy }} \sim \operatorname{Re}^{-1 / 4} v_{\text {th }}\end{array}$ & $\begin{array}{l}10^{6} \\
10^{5}\end{array}$ & $\begin{array}{l}10^{7} \\
10^{6}\end{array}$ \\
\hline \multicolumn{4}{|c|}{ Viscosities, $\mathrm{cm}^{2} / \mathrm{sec}$} \\
\hline $\begin{array}{l}\text { neutral viscosity } \\
\text { ion viscosity } \\
\text { magnetic (Spitzer) diffusivity }\end{array}$ & $\begin{array}{l}\nu_{n} \sim v_{\mathrm{th}}^{2} \tau_{n n} \\
\nu_{i} \sim v_{\mathrm{th}}^{2} \tau_{i i} \\
\eta \sim\left(k_{B} T\right)^{-3 / 2} m_{e}^{1 / 2} e^{2} c^{2} \ln \Lambda / 4 \pi\end{array}$ & $\begin{array}{l}10^{21} \\
10^{18} \\
10^{7}\end{array}$ & $\begin{array}{l}- \\
10^{26} \\
10^{4}\end{array}$ \\
\hline \multicolumn{4}{|c|}{ Dimensionless Numbers } \\
\hline $\begin{array}{l}\text { hydrodynamic Reynolds } \\
\text { magnetic Reynolds } \\
\text { magnetic Prandtl }\end{array}$ & $\begin{array}{l}\operatorname{Re} \sim v_{\mathrm{th}} L / \nu \\
\mathrm{R}_{m} \sim v_{\mathrm{th}} L / \eta \\
\operatorname{Pr} \sim \nu / \eta \sim\left(k_{\eta} / k_{\nu}\right)^{2}\end{array}$ & $\begin{array}{l}10^{5} \\
10^{19} \\
10^{14}\end{array}$ & $\begin{array}{l}10^{4} \\
10^{27} \\
10^{22}\end{array}$ \\
\hline
\end{tabular}


Table 2. Parameters of the SSF equation.

\begin{tabular}{cccccccccc}
\hline \hline \multirow{2}{*}{ Dimension } & Velocity Field & $a$ & $A$ & $B$ & $C$ & $s_{0}$ & $\xi_{0}$ & $\lambda_{0}$ & $\lambda_{\max }$ \\
\hline \multirow{2}{*}{$d=3$} & Incompressible & $-1 / 4$ & $1 / 5$ & $2 / 5$ & $4 / 5$ & $-1 / 2$ & $3 / 2$ & $3 / 4$ & $4 / 5$ \\
& Irrotational & 1 & $3 / 10$ & $3 / 5$ & $1 / 5$ & $-1 / 2$ & $3 / 2$ & $1 / 8$ & $1 / 5$ \\
\multirow{2}{*}{$d=2$} & Incompressible & $-1 / 3$ & $1 / 4$ & $-1 / 4$ & 0 & 1 & 2 & $-1 / 4$ & $-3 / 16$ \\
& Irrotational & 1 & $3 / 4$ & $5 / 4$ & 0 & $-1 / 3$ & $2 / 3$ & $-1 / 12$ & $5 / 48$ \\
\hline
\end{tabular}

